\title{
Die Partikeln schon und noch in der linearen Abfolge mit Temporaladverbien
}

\author{
Anna Kutscher (Bielefeld)
}

\begin{abstract}
The particles schon and noch in German add an aspectual reading to events in different assertions. While schon tends to a perfective reading of an event, an imperfective reading appears with noch, hence both particles have a similar function on the event modification. Overtly, they are located unrestricted in the middle field of a German clause and can freely change their position towards temporal adverbs such as heute, jetzt, bald etc. It seems that syntax does not have an impact to order schon heute or heute schon. This variation is of main interest of the paper: Is there a linear restriction of adverbs and schon/noch, and does a change in the output cause a reinterpretation? The paper presents main assumptions on the word order of aspectual particles and temporal adverbs from the semantic and the syntactic perspective. An experimental study on prosodic factors will complete the overview and its preferences for the linearization of the sequences in question. The result is that common categorical grammar is not able to account for all possible outcomes with respect to contextual preferences. Therefore, a more flexible grammar concept is needed to build a usage-based concept on word order variation for these functional elements.
\end{abstract}

\section{$1 \quad$ Einleitung}

Dieser Aufsatz verfolgt das Ziel, variable Abfolgen zwischen temporalen Adverbien und aspektuellen Partikeln schon und noch auf der Oberflächenstruktur wie in (1) dahingehend zu erfassen, ob es grammatikalische Faktoren gibt, die die Ordnung regeln oder ob hier freie Variation vorliegt.

(1a) Ich brauche die Daten am besten noch/schon heute.

(1b) Ich brauche die Daten am besten heute noch/schon.

Beide Varianten in (1) sind grammatikalisch korrekt, i. e. die Abfolge der beiden funktionalen Elemente ist nicht restringiert. Auch ein semantischer Unterschied zwischen (1a) und (1b) ist nicht eindeutig identifizierbar. Dennoch scheint intuitiv ein subtiler Unterschied zwischen beiden Sätzen vorzuliegen, der sie nicht als identisch klassifizieren lässt. Es stellt sich die Frage, welche Faktoren diesen Unterschied bedingen, wenn sowohl semantisch als auch syntaktisch keine eindeutigen Generalisierungen bezüglich der Abfolge und ihrer Interpretation erfasst werden können. Ein möglicher Faktor zur Präferenz der einen oder anderen Abfolge kann prosodisch motiviert sein. Denn (1a) ist rhythmisch ausgewogen und kann daher gegenüber (1b) prä- 
feriert werden. Oder es finden sich Verwendungskontexte, in denen (1b) eine passendere Abfolge darstellt, bspw. bei einem verstärkten Akzent auf dem Adverb heute (hervorgerufen durch einen engen Fokus oder andere Faktoren). Diese beiden möglichen Faktoren (Rhythmizität und Fokusposition) werden experimentell getestet, inwiefern sie sich auf die Abfolge der Partikel und Adverbien auswirken.

Der Aufsatz wird zeigen, dass einerseits beide Varianten empirisch belegt sind, andererseits es eindeutige Präferenzen zu einem vorangestellten schon und einem nachgestellten noch gibt. Dies ist jedoch nur eine schwache Tendenz, denn abhängig vom Adverb kann sich die Präferenz ändern. Die traditionellen generativen Ansätze haben ein Problem mit der Ableitung solcher Strukturen, weil sie entweder zu restriktiv sind oder zu viel Variabilität zulassen.

Der Aufsatz ist folgendermaßen gegliedert: In Abschnitt 2 werden die beteiligten Komponenten wie Tempus, Aspekt und adverbiale Modifikation formal betrachtet, insbesondere die Annahmen zu schon und noch sowie ihr Beitrag zur Satzmodifikation. Als Ergebnis kommt heraus, dass die Modifikation der Aussagen durch Adverbien und Partikeln einen Einfluss auf den propositionalen Gehalt hat; die Relevanz der Oberflächenposition dieser Elemente im Satz bisher jedoch nicht betrachtet wurde.

Abschnitt 3 setzt sich mit den syntaktischen Analysen zu Abfolgen und Basispositionen von schon/noch und Adverbien auseinander. Wie bereits in Beispiel (1) zu sehen, erlaubt die Syntax den Elementen eine Variation in der Sequenzierung und stellt keine Restriktionen zur AdverbPartikel-Abfolge bereit. Hierbei geht es ausschließlich um die Oberflächenposition im Mittelfeld, da jegliche Bewegung ins Vor- oder Nachfeld funktional geprägt ist und weitreichenderen Faktoren unterliegt.

In Abschnitt 4 wird ein besonderer Blick auf die phonologische Komponente gerichtet. Da diese Grammatikebene die Outputform maßgeblich beeinflusst, werden einige Abfolge relevante Effekte zwischen Adverb und Partikel experimentell getestet. Die Ergebnisse zeigen, dass die phonologische Ebene an der einen oder anderen Stelle einen Einfluss auf die Output-Sequenz haben kann; jedoch ist er bei weitem nicht so stark, dass man die Variation darauf zurückführen kann.

Im letzten und abschließenden Abschnitt 5 wird die grammatische Einbettung der Beobachtungen zur Variation der Adverbien und Aspekt-Partikeln vorgeschlagen, die von einer regelhaften Ordnung der Elemente absieht. Wie es sich herausstellen wird, haben die Sprecher sowohl für die einzelnen Partikeln als auch für die Adverbien bestimmte Präferenzen. Frequenzbasierte und probabilistische Theorien stellen dabei eine geeignetere Alternative dar, mit besagten Daten umzugehen.

\section{Funktionale Aspekte der Adverbien und Partikeln}

\subsection{Aspekt und Tempus}

Bei der Auseinandersetzung mit schon und noch in Verbindung mit Adverbien gilt es als erstes zu bestimmen, mit welchen funktionalen Elementen hier umgegangen wird. Betrachtet man die modifizierenden Elemente in (2), so haben sie alle eine andere Funktion. Die Proposition (2a) wird mit dem Adverb (Adv) in (2b) temporal modifiziert: heute verortet die Handlung zeitlich 
in den Tag, an dem eine Äußerung erfolgt. Mit schon in (2c) kommt eine perfektive/punktuelle Komponente hinzu, in der die Proposition ein finales Ende erfährt: Die Daten werden seit einer Weile aber spätestens heute und nicht morgen benötigt. (2d) unterscheidet sich davon, da es durch noch eine imperfektive/durative Lesart bekommt: Man benötigt die Daten im Laufe des heutigen Tages.

(2a) Ich brauche die Daten.

(2b) Ich brauche die Daten heute.

(2c) Ich brauche die Daten schon heute.

(2d) Ich brauche die Daten noch heute.

Die Elemente heute, schon und noch haben somit folgende Funktionen: Temporaladverbien wie heute interagieren mit dem Tempus, indem sie den zeitlichen Rahmen einer Handlung innerhalb der ,universellen Zeit“ festlegen (cf. Guillaume 1964, Hewson 2012, Valin 1994). Dabei kann ein bestimmter Zeitpunkt im heutigen Tag gemeint sein oder der heutige Tag als Ganzes (cf. Rathert 2012). Die hinzukommenden Partikeln (Part) schon und noch tragen zur aspektuellen Dimension der Aussage bei. Aspekte sind nach Comrie (1976:3), different ways of viewing the internal constituency of a situation“ und geben zusätzliche Information zum perfektiven, imperfektiven, habituellen etc. Verlauf der geschilderten Ereignisse. ${ }^{1}$ Schon/noch sollen hier ihrer Funktion nach als Aspektpartikeln klassifiziert werden, weil sie einerseits zusammen mit dem Temporaladverb eine Konstituente bilden, die insgesamt die zeitliche Dimension der Aussage bestimmt. Andererseits unterscheiden sich die Partikeln prosodisch vom Adverb, da sie als Begleiter des Adverbs nicht den Primärakzent der Akzentdomäne tragen. Unabhängig von der Position vor oder nach dem Temporaladverb und unabhängig von der prosodischen Schwere (ein- bis mehrsilbig in (3)) des Adverbs befindet sich der Hauptakzent der Konstituente stets auf dem Adverb.

(3a) schon OFT/HEUte/geLEgentlich | OFT/ HEUte/geLEgentlich schon (3b) noch OFT/ HEUte/ geLEgentlich | OFT/ HEUte/ geLEgentlich noch

In Konstellationen wie (3) sind die Partikeln nicht betont und werden in gesprochenen Daten (Korpora- und Experimentaufzeichnungen) nicht selten reduziert ausgesprochen. In Anlehnung an Hall (1999:100-105) kann man sie als Funktionswörter klassifizieren, die als Klitika an das prosodische Wort des Adverbs angehängt werden und somit keinen eigenen Status als prosodisches Wort besitzen.

\subsection{Interpretation und Formen von schon/noch}

Die Betrachtung von schon und noch legt zunächst nahe, dass es sich hier um zwei Elemente handelt, die die Satzaussage bezüglich ihrer Perfektivität/Imperfektivität modifizieren. Während schon auf einen abgeschlossenen Vorgang der Äußerung bzw. einen eingetroffenen Zustand referiert, befindet sich dieser Vorgang in Verbindung mit noch weiterhin im Prozess.

(4) Schläft Peter?

(4a) Schläft Peter noch?

\footnotetext{
${ }^{1}$ Für ausführliche Analysen der Interaktion zwischen diversen Temporaladverbien, Tempus und Aspekt cf. u. a. Musan (2002).
} 
(4b) Ja, Peter schläft noch.

(4c) Nein, Peter schläft nicht mehr.

(4'a) Schläft Peter schon?

(4'b) Ja, Peter schläft schon.

(4'c) Nein, Peter schläft noch nicht.

(Doherty 1973:154)

Doherty (1973) geht davon aus, dass beide Partikeln die Fähigkeit haben, den Sachverhalt einer Aussage in zwei Phasen zu teilen. Dabei unterscheiden sie sich in der Festlegung der Präsupposition auf eine dieser Phasen. Während noch in einem Satz $\left(\mathrm{S}^{2}\right)$ in (4) die Präsupposition hat, dass die Aussage ( $\mathrm{S}=$ Peter schläft) auch in der vorangegangenen Phase stattgefunden hat, ist die gleiche Proposition in (4') in Verbindung mit schon so gestaltet, dass der Sachverhalt (S) in der Phase zuvor nicht gegeben war, also nicht-S ist (Doherty 1973:154). Man kann es schematisch wie in Abb.1 darstellen, dass beide Phasen der geäußerten Handlung innerhalb eines Zeitrahmens liegen, die Präsupposition sich jedoch darin unterscheidet, dass in Phase ${ }_{1}$ der Sachverhalt $\mathrm{S}$ bereits angenommen wird (noch) oder nicht (schon).

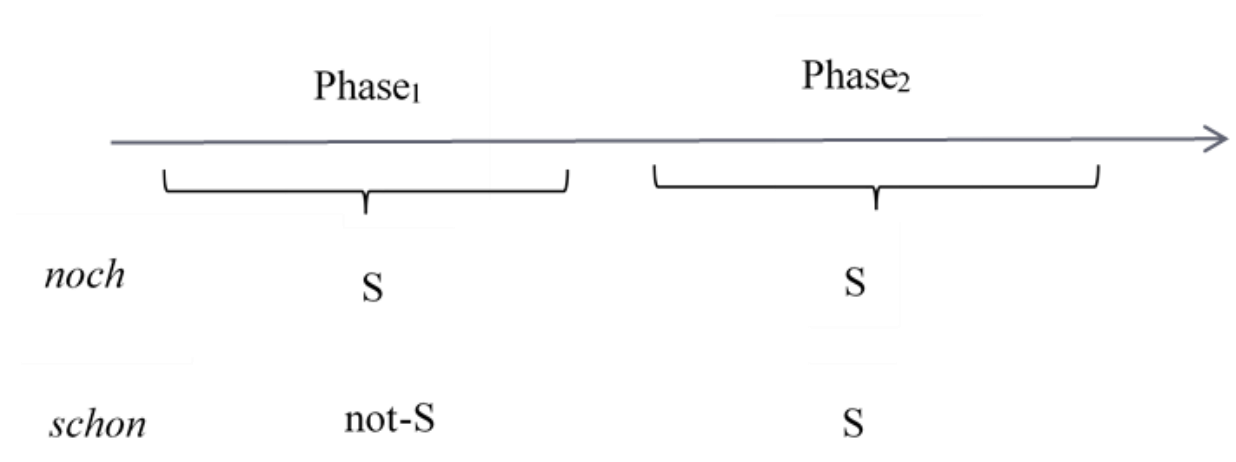

Abbildung 1: Propositionale Phasenordnung der Aussage mit schon/noch

König (1977) erweitert diese Unterteilung, indem er eine weitere Phase hinzufügt. In seinem Ansatz besteht die Präsupposition einer Äußerung aus einer Abfolge von zwei Phasen: $<$ S, not$\mathrm{S}>$ für noch und <not-S, S> für schon. Konkret bedeutet es, dass mit der Äußerung (4a) der Sprecher einerseits den Sachverhalt des Schlafens presupponiert, andererseits aber auch, dass danach das nicht-schlafen eintreten wird. In (4'a) ist die Abfolge der präsupponierten Phasen umgekehrt: erst wird vorausgesetzt, dass es einen Zustand des nicht-Schlafens gibt und daraufhin das Schlafen folgt. Beide Partikeln sehen also so aus, dass sie die distinktive Aufgabe innehaben, eine Aussage in unterschiedliche Phasen zu gliedern und daraus verschiedene Präsuppositionen abzuleiten.

Die Analysen von Doherty und König behandeln in erster Linie die lexikalische Semantik von schon/noch. Entsprechend scheint es keinen Unterschied zu geben, wenn die Oberflächenpositionen zwischen Adverb und Partikel vertauscht werden. Analysiert man das Beispiel (1), hier wiederholt als (5) und (5'), nach dem Modell von Doherty oder König, kommen identische Resultate zustande.

${ }^{2} \mathrm{~S}$ steht in Dohertys Notation für den Sachverhalt der im Satz ohne schon/noch ausgedrückt wird. 
(5) Ich brauche die Daten schon heute.

(5') Ich brauche die Daten heute schon.

Proposition: Phase1 (not-S), Phase3 (S)

Assertion: Phase2 (S)

Die Aussage in (5) bzw. (5') präsupponiert, dass es eine Phase des Ereignisses gibt, wo nicht-S gilt (= Ich brauche die Daten noch nicht/nicht heute) und darauf eine Phase mit S (= Ich brauche die Daten schon heute) folgt (in umgekehrter Abfolge für noch). Es macht daher keinen Unterschied, in welcher Abfolge das Temporaladverb und die Partikel zueinanderstehen. Anders formuliert: Die Semantik der hier betrachteten Adverbien und Partikeln, wie von Doherty und König angenommen, gibt keinen Anlass dazu, ihre variable Kombination unterschiedlich zu interpretieren.

Beide Partikeln, sowohl schon als auch noch, haben zunächst die gleiche Funktion, ${ }^{3}$ eine Relation der Zustände/Ereignisse zu einem früheren und einem späteren Zeitpunkt anzugeben:

„[...] whereas a simple sentence asserts (or asks, or commands) something about a particular situation, a sentence with noch or schon relates this assertion to the "same" topic situation at some earlier or later time. [...] both schon and noch make the sentence "look to the left AND to the right".

(Klein 2006:17f.)

\footnotetext{
${ }^{3}$ Neben der Funktion der Phasenbeschreibung erwähnt Doherty auch die evaluative Funktion beider Partikeln. So drückt der Sprecher mit der Verwendung von schon ein Eintreten des Zustandes ein, der früher als erwartet kommt. Noch steht dabei für die Sprecherhaltung, nämlich, dass die erste Phase länger/intensiver/etc. dauert als angenommen. König (1977) merkt dazu an, dass insbesondere die akzentuierten Formen von schon und noch die evaluative Lesart hervorrufen. Dabei sind die Partikeln schon und noch nicht ausschließlich temporal zu interpretieren, sondern auch auf andere skalare Dimensionen (Länge, Qualität, ...) übertragbar.
}

Temporal:

Noch ist Paul kleiner als Peter.

Evaluativ, nicht temporal:

Paul ist nòch größer als Peter.

Peter ist groß. Aber Paul ist nòch größer (als Peter). Paul ist größer noch als Peter.

(König 1977:188)

Eine Besonderheit in der kontextuellen Verwendung stellt schon dar, das im Vergleich zu noch auch als Fokuspartikel oder Modalpartikel ohne Aspektsemantik interpretiert werden kann (cf. dazu Féry 2010, König 1977, Ormelius-Sandblom 1996).

Fokuspartikel:

Schon der Gedanke an Paul macht Peter verrückt.

Der Vorschlag muss schon deshalb abgelehnt werden, weil er nicht detailliert ausgearbeitet worden ist.

(König 1977:186)

Modalpartikel:

Wir werden das Geld schon finden.

Das ist schon war, aber ....

(König 1977:173)

Diese Verwendung als Fokus- und Modalpartikel, so interessant sie auch sein mag, soll hier nicht weiter thematisiert werden. Wie bereits erwähnt, stehen die Partikelformen im Vordergrund, die als funktionale Einheiten mit einem Adverb eine aspektuelle Lesart tragen und als prosodische Klitika mit dem Adverb eine Akzentdomäne bilden. Daher wird auf die Analyse der akzentuierten Partikeln und ihre Distribution im Satz verzichtet. 
Dadurch, dass die Partikeln die gleiche Funktion haben, ist zu erwarten, dass ihre Kombination mit anderen Kategorien sowie die Positionen auf der Oberfläche sich nicht wesentlich voneinander unterscheiden. Dass diese Annahme nur bedingt zutrifft und andere Faktoren (syntaktisch, prosodisch) eine Auswirkung darauf haben, ist Thema der folgenden Abschnitte.

\section{$3 \quad$ Kombination von funktionalen Elementen}

Während es im vorherigen Abschnitt um die lexikalische Semantik der Partikeln schon/noch ging und ihr funktionaler Beitrag zur Propositionen im Vordergrund stand, soll es im Folgenden um die Kombination der Partikeln mit anderen funktionalen Kategorien gehen. In der älteren Forschungsliteratur lassen sich diverse Vorschläge für die Kompatibilität oder auch Inkompatibilität der Partikeln mit bestimmten Tempora oder Adverbien finden. So argumentieren Hoepelman/Rohrer (1981) im Vergleich zu vorher skizzierten Ansätzen von Doherty (1973) und König (1977) dafür, dass die Interpretation von schon/noch nicht intrinsisch aus den Partikeln herausgelesen werden kann, sondern anhand der semantischen Verbtypen und der Sprechererwartung bestimmt wird. Sie führen an, es sei nicht möglich, noch in Verbindung mit Achievement-Verben zu verwenden, ohne dass dabei die iterative (6b) oder andere Lesarten entstehen. Accomplishment-Verben (6a) sind hingegen nicht an iterative Interpretationen gebunden.

(6a) Hans schlief noch.

(6b) *Die Bombe explodierte noch.

(Hoepelman/Rohrer 1981:105)

Aber auch in der Verbindung mit Activity-Verben haben die Partikeln unterschiedliche Interpretationen. So gibt es keinen Rückschluss darauf, dass der Gebrauch von schon in Kombination mit durativen Verben eine Gleichzeitigkeit von Sachverhalten ausdrückt (siehe 8a und 8b). In Kombination mit punktuellen Verben ist es jedoch so, dass beide Sachverhalte gleichzeitig stattfinden (7c und 7d). Die Autoren nehmen hierfür ein zweites schon an. $^{4}$

(7a) Als die Oper anfing, schlief Hans schon.

(7b) Die Oper fing an und schon schlief Hans.

(7c) Als die Oper anfing, explodierte die Bombe schon.

(7d) Die Oper fing an und schon explodierte die Bombe.

(Hoepelman/Rohrer 1981:108)

Die unterschiedliche Kombinationsfähigkeit der Partikeln mit punktuellen und durativen Verben führt in Hoepelman/Rohrers Analyse zu Auswirkungen in Bezug auf die Ordnungsrestriktionen zwischen der Partikel und dem T(empus)-Kopf, die empirisch nicht haltbar sind. So ist sei bspw. nicht möglich, noch mit Perfekt zu verwenden. Das Argument gegen Sätze wie (8) ist, dass das Perfekt im Deutschen Eigenschaften von Subjekten in der Vergangenheit beschreibt, die auch in der Gegenwart Bestand haben (Hoepelman/Rohrer 1981:113); (8b) zieht also immer (8c) nach sich. So kommt es zu einem logischen Widerspruch, wenn das Perfekt

\footnotetext{
${ }^{4}$ Ähnliche Unterschiede gibt es auch für noch ${ }_{l}$ und $n o c h_{2}$, auch sie führen zu verschiedenen Interpretation bzw. Kombinationsmöglichkeiten mit entsprechenden Verbklassen.
} 
Zustände beschreibt, die weiterhin gelten und mit einer Partikel wie noch kombiniert wird, deren Präsupposition es ist, dass die Phase solcher Zustände (S) endlich und ein nicht-S als Folge zu erwarten ist. Daher lassen Sätze wie (8) nur evaluative oder additive Lesarten von noch zu.

(8a) *Hans hat noch gegessen.

(8b) Hans hat gegessen.

(8c) Hans ist einer der aß. [sic.]

(Hoepelman/Rohrer 1981:113)

Allerdings lassen sich in Korpora diverse Belege finden, die genau die erwähnte Inkompatibilität enthalten, wie in (9a) und (9b).

Parallel zum Perfekt betrachten Hoepelman/Rohrer die Kombination von noch mit Futur: die Abfolge $\left[T_{F u t}\right.$ noch] stellt für die vorgeschlagene Analyse kein logisches Problem dar. Dadurch, dass die Phasen von noch als Relation $<$ S, not-S $>$ abgebildet sind, und der Geltungsbereich der Handlung in der Zukunft liegt, liegt auch der erwartete nicht-Zustand in der Zukunft. Problematisch wird es mit der umgekehrten Abfolge [noch $\left.\mathrm{T}_{F u t}\right]$ : Die Partikel legt die Phasen so fest, dass not-S dann zu erwarten ist, wenn $\mathrm{T}_{\text {Fut }}$ applizieren soll. Beide Elemente fordern daher widersprüchliche Zustände innerhalb der gleichen Phase, noch den not-S-Zustand und $\mathrm{T}_{\mathrm{FUT}}$ den S-Zustand. Auch hier existieren Belege wie (9c) und (9d), die die Annahme widerlegen. ${ }^{5}$

(9a) Silvester hat das VW-Motorsportteam noch gefeiert, morgen wird es ernst. (Braunschweiger Zeitung, 02.01.2009).

(9b) Eine kleine Bevölkerungsgruppe des Dörfchens freilich hat sich jenen fürstlichen Hochzeitstag durch mündliche Überlieferung noch lebendig in Erinnerung bewahrt. (Braunschweiger Zeitung, 03.01.2009).

(9c) Ein Sprecher der Staatskanzlei wies am Sonntag darauf hin, dass Hirche nach der Amtsübergabe zunächst noch als Vertreter des Landes in den Aufsichtsräten von VW und Messe tätig sein wird. (Hannoversche Allgemeine, 19.01.2009: 7).

(9d) Im Lauf der Zeit sollen sich 95 Hektar Rasen mit Quadern und diese wiederum mit leicht und mittelschwer verseuchtem Material füllen, das noch in 300 Jahren radioaktive Strahlung freisetzen wird. (Hannoversche Allgemeine, 07.02.2009: 3).

Ein Versuch, Kombinationen der Partikeln in ihrer Modifikation der Aspektualität mit entsprechenden Verbklassen und Tempora zu erfassen schlägt also fehl. Viel mehr sind es lexikalische Beschränkungen wie die Aktionsarten der Verben und Tempora, in denen die Verben eingesetzt werden, die eine Interpretationsänderung hervorrufen. Generalisierungen, wie sie bspw. von Hoepelman/Rohrer vorgenommen werden, lassen sich empirisch schnell widerlegen.

Eine der ausführlichen semantischen Auseinandersetzungen mit den Partikeln schon und noch in Kombination mit Zeitadverbialen findet sich in Löbner (1989). Löbner geht davon aus, dass es vier Typen dieser Partikeln und ihrer Verwendung gibt: Einerseits als Operatoren auf dem

\footnotetext{
5 Ähnliche Inkompatibilitäten zwischen schon/noch und bestimmten Tempora nimmt auch Rathert (2012) an. Eine Annahme ist, dass die temporale Kombination schon immer mit Plusquamperfekt und Perfekt funktioniere, jedoch nicht mit Präteritum. Auch hierfür finden sich zahlreiche Belege in Korpora der gesprochenen und geschriebenen Sprache, die die Behauptung widerlegen.
} 
Satzfokus (Typ 1) oder dem engen Fokus (Typ 2) von imperfektiven Sätzen. Andererseits stellen sie einen fokussierenden Operator auf temporale Adverbien in perfektiven (Typ 3) oder imperfektiven (Typ 4) Sätzen dar. Für die vorliegende Fragestellung sind nur die beiden letzten Typen relevant, in denen die Partikeln einen Interpretationsrahmen für Adverbien bilden. In Löbners Analyse bewirken die Partikeln eine skalare Interpretation des Adverbs, die sich je nach Kontext zeitlich, nummerisch, adjektivisch etc. äußern kann (cf. 10).

(10a) Er kommt schon morgen in Frankfurt an.

(10b) Schon vor drei Tagen wurde sie krank.

(Löbner 1989:193)

So lässt sich das Adverb morgen in (10a) auf einer Skala abbilden, die zumindest die Bereiche ,morgen“ und ,nicht morgen' hat. Im Fall von (10b) kann das Adverbial vor drei Tagen eine nummerische Skala beschreiben (,nicht vor zwei Tagen', ,nicht heute ). Ebenso kann (10b) aber auch eine wertende Skalierung beinhalten (, es ist nicht so lange her und daher ist es verständlich, dass sie krank ist 'oder, es ist sehr lange her, normalerweise sollte sie nicht mehr krank sein').

Unabhängig davon, welcher Art die skalare Interpretation für das Adverb durch eine Partikel ausfällt, trägt die Partikel dazu bei, dass in Sätzen wie (10) die Präsupposition „, The event e takes place at some time $T^{\prime \prime}$ entsteht (cf. Löbner 1989). I. e. dass das vom Satz ausgedrückte Ereignis durch die Partikel schon in zwei Teiläußerungen gespalten wird: eine Zeit, in der das Ereignis stattfindet und eine, wo es nicht der Fall ist. Der Kontext ist dabei ausschlaggebend dafür, wie die perfektive Lesart der Partikel zu verstehen ist: Je nach Erwartungshaltung des Sprechers oder Hörers kann das Ereignis $e$ früher oder später eintreten: Er kommt schon morgen in Frankfurt an (Er wurde aber erst nächste Woche erwartet). Löbners Analyse hat in der Folgediskussion diverse Vorschläge zur Betrachtung von schon und noch ausgelöst (cf. u. a. Mittwoch 1993, van der Auwera 1993, Löbner 1999). Insbesondere dahingehend, ob die kompositionelle Interpretation der Partikeln und der Proposition so aufrechterhalten werden kann wie von Löbner (1989) vorgeschlagen. Es sieht ganz danach aus, dass die lexikalische Betrachtung der einzelnen Partikeln nicht ausreicht, um die Interpretationsdimension der Partikeln zusammen mit anderen funktionalen Kategorien wie Tempus, Aktionsart etc. erfassen zu können. Viel mehr sind pragmatische Faktoren dafür ausschlaggebend, wie die Partikel zusammen mit der Proposition zu interpretieren ist.

Während es also in der älteren Betrachtung von schon und noch lediglich um die lexikalische Semantik und die Komposition der einzelnen Bedeutungseinheiten beider Partikeln mit den semantischen Eigenschaften der Adverbien oder ganzer Propositionen ging, richten sich neuere Ansätze zur Kombination solcher funktionalen Einheiten eher am Kontext einer Äußerung aus. Modelle wie von Farkas und Bruce $(2010)^{6}$ folgen dabei dem Ansatz der dynamischen Bedeutungsinterpretation, bei der der lexikalische Inhalt nicht allein dem Lexem, sondern auch seiner kontextuellen Einbettung entnommen wird (bspw. Stalnaker 2002, Büring 2003, Gunlogson 2003). Dabei werden neben der Komposition auch andere Mechanismen wie die Koerzion angenommen, die dazu beitragen, eine endliche Menge von lexikalischen Einheiten in unendlich

6 Übernommen bspw. von Müller (2018) zur Analyse von Kombinationen von Modalpartikeln im Deutschen. 
vielen Situationen einzusetzen und so in einem spezifischen Kontext entsprechende Interpretationen zu schaffen (cf. bspw. Pustejovsky 2011, Blumfeld 2011). Für die Interpretation der Partikeln und der Adverbien bedeutet es also, dass sie in den entsprechenden Kontexten in Kombination mit der Aktionsart des Verbs, dem Tempus und weiteren Merkmalen nicht nur mit dem perfektiven oder dem imperfektiven Aspekt verbunden sind, sondern andere Lesarten zulassen (skalar, iterativ, evaluativ etc. siehe Fußnote 3). Umfangreiche Erkenntnisse, inwiefern die Abfolge der einzelnen Bestandteile zueinander eine solche Interpretationsmodifikation steuert liegen für die hier betrachteten Fälle von schon/noch und Temporaladverb noch nicht vor.

Zusammenfassend lässt sich sagen, dass die Partikeln schon und noch in der aspektuellen Lesart unterschiedlich mit anderen Kategorien kombiniert werden können: In Verbindung mit unterschiedlichen Verbklassen können Re-Interpretationen, wie eine iterative Lesart, entstehen, diese sind jedoch optional. Bezüglich der Kombination mit dem Tempus lassen sich empirische Belege finden, die vorgeschlagene Inkompatibilitäten mit dem einen oder anderen Tempus widerlegen. In der Kombination mit Temporaladverbialen fügen die Partikeln eine skalare Interpretation hinzu, die kontextuell erschlossen werden muss. Diese Skala muss nicht notwendigerweise immer mit schon als perfektiven und noch als imperfektiven Aspekt angegeben werden. Viel entscheidender ist dabei, welche anderen semantischen und pragmatischen Faktoren die Skala bestimmen. Inwiefern die Reihenfolge der Partikel und des Adverbs hierfür ausschlaggebend ist, kommt bei den Analysen jedoch nicht heraus. Man kann also nicht per se sagen, dass [Adverb Partikel] eine andere Interpretation nach sich zieht als [Partikel Adverb]. Semantisch betrachtet gibt es deshalb keine Restriktionen bezüglich der Abfolge beider Elemente.

\subsection{Oberflächenabfolgen und Skopus}

Während die bisher skizzierten Arbeiten keine Hinweise darauf geben, dass die Abfolge der funktionalen Kategorien wie Adverbien und Partikeln Interpretationsunterschiede hervorrufen kann, gehen andere Autoren davon aus, dass durch die lineare Präzedenz die Skopusrelationen zwischen den Elementen abgebildet werden. Insbesondere die Anhänger der Tradition von spurenbasierten Bewegungsoperationen für Scrambling legen eine Abhängigkeit zwischen Struktur und Interpretation zugrunde (cf. u. a. Fanselow 1990; Frey 1993; Hinterhölzl 2006; G. Müller 1995; Sabel 2005). Unterschiedliche Abfolgen innerhalb der Struktur gehen hier mit unterschiedlichen Fokusprojektionen und Skopusresolutionen einher und bringen Markiertheitsunterschiede mit sich. In der Regel befassen sich Scramblingtheorien mit Abfolgevariationen zwischen mehreren Konstituenten. Der hier betrachtete Fall zwischen Adverbien und Partikeln skizziert aber die Abfolgevariation innerhalb einer Konstituente und ist daher nicht identisch mit Scramblingphänomenen. Aus diesem Grund können mögliche angenommene Filter, die Scrambling auslösen, ebenfalls für die Variation der Adverb-Partikel-Sequenz verantwortlich sein oder sich gänzlich von diesen unterscheiden. Eine der umfangreichsten und am häufigsten diskutierten Analysen zur linearen Abfolge von funktionalen Kategorien ist in Cinque (1999, 2004, 2014) vorgeschlagen. ${ }^{7}$ Cinque geht davon aus, dass die syntaktische Struktur eine Vielzahl an funktionalen Kategorien innehat, in denen die entsprechenden Elemente basisgeneriert

\footnotetext{
${ }^{7}$ Für Kritik und Gegenevidenzen cf. u. a. Ernst (2002), Haider (2000), Rosengren (2000) und andere Autoren.
} 
werden. Auch wenn diese Kategorien bei Cinque syntaktisch verankert werden, sind sie in ihrem Kern semantischen Ursprungs und bilden sowohl die Ordnung von Affixen als auch entsprechende Konstituenten in einer universellen Basisabfolge ab. Die Kernidee davon ist, dass von der Basisstruktur abweichende Abfolgen unterschiedliche Interpretationen nach sich ziehen. Diese werden erreicht, indem neben der Basisgenerierung weitere Bewegungsprozesse von Konstituenten vollzogen werden.

Überträgt man die Hierarchie auf die hier relevanten Paarungen ${ }^{8}$ von Temporal-Adverbien und aspektuellen Partikeln schon und noch, bedeutet es, dass je nach Einstufung der semantischen Funktion der Köpfe noch und schon unterschiedliche Basisabfolgen zu erwarten sind. Nimmt man also an, dass die unmarkierte Interpretation der Partikeln von noch Aps kontinuative $_{\text {und von }}$

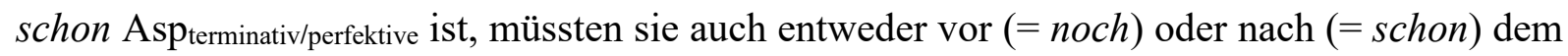
habituellen Aspekt oder dem Tense-Kopf der Adverbien realisiert werden. ${ }^{9}$ Anders formuliert: die Sequenzen immer schon, immer noch sowie jetzt schon und heute noch sind als unmarkierte Abfolgen innerhalb der Hierarchie abgebildet. Ihre Umstellungen zu schon immer, noch immer etc. stellen hingegen abgeleitete Abfolgen dar und sollten eine andere, markierte Interpretation nach sich ziehen. Ähnliches sollte für das proximative bald gelten. Zu erwarten ist hier eine Basisposition nach dem terminativ/perfektiven Aspekt der Partikel. Schon bald (12a) wäre daher unmarkiert und bald schon (12b) als abgeleitet zu betrachten. Darüber, ob die Satzpaare in (11) und (12) tatsächlich unterschiedlich interpretiert werden, lässt sich streiten. Die Proposition, die den Paaren in (11) und (12) zugrunde liegt, ist identisch. In den Paaren wird der zeitliche Rahmen der Aussage auf die Zeitangabe des Adverbs begrenzt, sowie die Referenzzeit als nicht abgeschlossen durch noch oder abgeschlossen durch schon angegeben.

Tendenziell sind die Adverbien in der [Adv Part]-Abfolge mit einer stärkeren prosodischen Prominenz und somit einem emphatischeren Nachdruck versehen, auch wenn hier entsprechende Kontexte fehlen. Ein solcher Nachdruck ist jedoch auch in der umgekehrten Abfolge möglich. Dass sich die Umstellung der Elemente von der Basisabfolge in eine markierte Sequenz auf die Interpretation auswirkt, kann jedoch nicht bestätigt werden; dafür sind die Unterschiede zu gering (ein emphatischer Akzent auf dem Adverb ist bspw. sowohl mit einer vorangestellten als auch einer nachgestellten Partikel möglich).

(11a) Wir haben immer schon hier gewohnt.

Wir haben schon immer hier gewohnt.

(11b) Paul trägt immer noch die Sonntagszeitung aus.

Paul trägt noch immer die Sonntagszeitung aus.

(11c) Ich habe jetzt schon keine Lust mehr.

Ich habe schon jetzt keine Lust mehr.

(11d) Ich rufe heute noch beim Tierarzt an.

Ich rufe noch heute beim Tierarzt an.

(12a) Der Junge wird schon bald die Schule beenden.

\footnotetext{
${ }^{8}$ Für die hier diskutierte Analyse wurden folgende Paare verwendet: immer schon, immer noch, jetzt schon, heute noch, bald schon, oft schon. Siehe dazu auch Abschnitt 4.2.1

${ }^{9}$ Danke an einen anonymen Reviewer für den Hinweis, dass in anderen Kontexten auch die Zuordnung der funktionalen Kategorie unterschiedlich ausfallen kann. Die im Experiment verwendeten Stimuli (siehe Anhang) sind jedoch mit Hinblick auf die hier notierten Funktionen konstruiert.
} 
(12b) Der Junge wird bald schon die Schule beenden.

(12') Wann wird Peter mit der Schule fertig?

(12'a) Der Junge wird [schon BALD] die Schule beenden.

(12'b) Der Junge wird [BALD schon] die Schule beenden.

Ein zweiter Ansatz, bei dem die Oberflächenposition der Partikel Skopus-relevant ist, kommt von Klein (2006). Die Position der Partikel ist in seiner Analyse entscheidend, weil die Äußerung dadurch in zwei Bereiche aufgespalten wird: $\alpha^{10}$ und $\omega$. Beiden Bereichen stehen $\alpha^{\prime}$ und $\omega^{\prime}$ gegenüber, die als eine alternative Proposition zur Äußerung als identisch oder minimal unterschiedlich ausfallen kann. $\alpha$ und $\alpha^{\prime}$ geben dabei die Situation wieder, die mit einem früheren (noch) oder späterem (schon) Zeitpunkt verglichen wird. $\omega$ und $\omega^{\prime}$ stehen für die möglichen kontextrelevanten Aspekte. Die Position der Partikel vor dem Adverb gibt an, dass nicht nur eine ,andere“ Handlung, sondern außerdem die relevante Zeit in einer zusätzlichen Proposition geäußert wird. Eine Position der Partikel nach dem Adverb lässt die Zeit unverändert, sodass die alternative Proposition nur noch in der Handlung ausgedrückt wird.

Diese Festlegung ist allerdings irreführend, weil hier nicht nur die Position der Partikel sondern auch ihre prosodische Einbettung ausschlaggebend sind. Betrachtet man (12b), können zwei Phrasierungsalternativen wie in (13) entstehen. Für (13b) trifft Kleins Vorhersage zu, dass die Äußerung ein $\omega$ (= die Schule beenden) eine mögliche Alternative $\omega^{\prime}$ (= die Ausbildung anfangen o. ä.) präsupponiert. Schon fungiert hier als Fokuspartikel zur VP. Kleins Vorhersage trifft jedoch nicht zu, wenn schon in der Akzentdomäne (AD) des Adverbs wie in (13a) enthalten ist und somit eine Fokuspartikel zum Adverb ist. Eine Betrachtung der Oberflächenposition ohne entsprechende phonologische Phrasierung reicht somit nicht aus, weil auch eine nachgestellte Partikel die temporale Angabe in $\omega^{\prime}$ einschließen kann.

(13a) Der Junge wird (bald schon) AD (die Schule beenden) AD.

(13b) Der Junge wird (bald) AD (schon die Schule beenden) AD.

Unklar bleibt außerdem in Kleins Ansatz, wie übrigens auch bei Cinque, welche Faktoren die Umstellung beeinflussen, bzw. welche Merkmale die Bewegung von Adverbien und aspektuellen Partikeln auslösen. Klassische Trigger wie Kasus oder Skopus sind für die Adverbien und Partikeln irrelevant bzw. bringen keine Unterschiede in der Interpretation. Auch Bindung und Referenz stellen keine geeigneten Auslöser dar. Warum es also die Variabilität in der AdverbPartikel-Sequenz im Mittelfeld gibt, ist anhand der generativen Analysen nicht erklärt.

Eine bessere Alternative versprechen Ansätze, die für variable Oberflächenstrukturen nicht von einer Basisabfolge und Bewegungsoperationen ausgehen und stattdessen wettbewerbsorientiert mehrfache Faktoren für die Outputform heranziehen (cf. Struckmeier 2014; Wurmbrand 2008). Da auch diese Ansätze sich mit der Umstellung von Konstituenten und nicht auf konstituenteninterne Variation beziehen, fehlen hier entsprechende Trigger, die über die Skopusbetrachtung hinausgehen. Informationsstrukturelle Aspekte wie die Bewegung ins Vorfeld oder Nuklearakzent-bedingte Umstellungen am rechten Rand der Intonationsphrase wären ein möglicher Faktor für die Variation, beträfen aber auch hier die Konstituente als Ganzes.

\footnotetext{
${ }^{10} \alpha$ steht für alle Elemente, die nicht im Skopus der Partikel eingeschlossen sind; $\omega$ für alle Elemente, über die die Partikel Skopus nimmt.
} 
Ein weiteres Problem für die Analyse stellen außerdem theorieinterne Limitierungen dar: Ein klassischer Fall für eine outputorientierte Syntaxtheorie ist die Optimalitätstheorie, die Beschränkungen auf unterschiedlichen linguistischen Ebenen formulieren kann (cf. bspw. Choi 1996, Müller 1999 für Scrambling-Analysen) und daher die eine oder andere Adverb-PartikelSequenz im Wettbewerb potenziell als den Gewinner herausstellen kann. Allerdings erlaubt sie klassischerweise keine mehrfachen optimalen Kandidaten, die für die hier diskutierten Fälle notwendig wären.

Zusammenfassend lässt sich sagen, dass die vorgeschlagenen Unterschiede in der Abfolge der Adverbien und Partikeln als funktionale Kategorien keine Skopusunterschiede hervorbringen. Sowohl [Adverb Partikel] als auch [Partikel Adverb] sind als Abfolgen zulässig, ohne dass dabei unterschiedliche Auflösungen der Skopusbeziehungen notwendig sind. In beiden Abfolgen ist es möglich eine identische und neutrale Zeit-Aspekt-Lesart zu haben. Eine Ordnung der Elemente auf der Oberflächenform ist somit weder semantisch noch syntaktisch restringiert.

Gleichzeitig ist es so, dass man als Sprecher des Deutschen bei allen Beispielen subtile Präferenzen zu der einen oder anderen Abfolge hat. Diese können rein stilistischer Natur sein, die man grammatikalisch so nicht erfassen kann, oder aber phonologisch begründeten Parametern unterliegen. Einige dieser Parameter sollen deshalb im Folgenden diskutiert und auf ihr Potenzial der Einflussnahme zur Ordnung der Adverbien und schon/noch getestet werden.

\section{$4 \quad$ Phonologische Faktoren}

\subsection{Heaviness-Effekte}

Dass nicht immer allein die syntaktisch gesteuerten Prozesse für die Umstrukturierung der Wortabfolgen im linearen Output ausschlaggebend sind, sondern auch Prozesse der phonologischen Form die Anordnung der Konstituenten verändern können, soll hier kurz skizziert werden.

Das erste Beispiel für die Umstellung der Konstituenten auf der Oberfläche bezieht sich auf die phonologische Schwere und morpho-syntaktische Komplexität von einzelnen Elementen. Evidenzen dafür, wie diese Faktoren die Wortstellung beeinflussen, lassen sich in unterschiedlichen Phänomenen beobachten. Die bekanntesten Ansätze hierfür sind womöglich Behaghels (1932) “Gesetz der wachsenden Glieder” oder das LIPOC-Prinzip nach Dik (1978) und darauf basierte spätere Analysen zu Heaviness-Effekten.

Beide Formulierungen, bei Behaghel aufgrund der Länge und bei Dik aufgrund der Komplexität, führen dazu, dass eine unmarkierte Wortstellung zugunsten einer markierten Abfolge aufgegeben wird, indem die Konstituenten sich von kurz nach lang bzw. von einfach nach komplex ausrichten, ohne dass sich dabei andere Interpretationen ergeben. Im Deutschen lässt sich ein solcher Effekt unter anderem in der Versetzung von Nebensätzen oder Präpositionalphrasen ins Nachfeld demonstrieren (14).

(14a) Wir in Niedersachsen müssen erkennen, dass unser Schicksal ganz entscheidend von der Politik t abhängt, die in Europa gemacht wird. ${ }^{11}$

${ }^{11}$ HAZ09/JAN.00076 Hannoversche Allgemeine, 02.01.2009: 2 
(14b) Das würde auch schwierig werden mit einem Präsidenten $t$ als Staatsoberhaupt, der ausgewiesener EU-Skeptiker ist. ${ }^{12}$

Aus den Beispielen in (14) kann man ablesen, dass die Standardabfolgen der Konstituenten zugunsten einer Abfolge aufgegeben werden, in der längere Konstituenten vorwiegend am rechten Rand des Satzes realisiert werden. Man kann daher sagen, dass „,im Deutschen [...] kurze Konstituenten bevorzugt vor langen Konstituenten platziert werden“ (Primus 1994:40).

Auch wenn es plausible Beispiele dafür gibt, dass die prosodische Schwere Auswirkungen auf die Oberflächenpositionen von Konstituenten haben kann, lässt sich diese Annahme im Fall der Adverb-Partikel-Kombination nicht aufrechterhalten. Die Partikeln schon/noch unterscheiden sich in ihrer Länge bzw. Komplexität wenig von den betrachteten Adverbien (immer, heute, jetzt, bald, oft); beide Wortarten sind überwiegend einsilbig bzw. wie im Fall von heute reduzierbar auf heut'. Dass eine finale Schwa-Silbe in immer sich auf eine präferierte Voranstellung der Partikel auswirkt, ist auch wenig plausibel. Somit kann die prosodische Schwere als Kriterium für die lineare Abfolge der betrachteten Sequenzen ausgeschlossen werden.

\subsection{Rhythmizität und Basisabfolgen auf der Oberfläche}

Ein weiterer prosodisch motivierter Faktor für die Umstellung von Konstituenten im linearen Output ist die rhythmische Verteilung der Tonakzente innerhalb von Intonationsphrasen. Je nach Position der Partikel kann man einen durchgehenden Rhythmus (Daktylus in 15a) oder einen rhythmischen Bruch (15b) erreichen.

(15a) Ich brauche die Daten am besten noch heute, damit der Bericht morgen früh fertig ist.

(15b) Ich brauche die Daten am besten heute noch, damit der Bericht morgen früh fertig ist.

Arbeiten von Shih et al. (2015), Vogel et al. (2015), Kentner (2017), Shukla/Nespor (2010), Kutscher (2016) legen nahe, dass die rhythmische Ausrichtung von Sätzen einen erheblichen Einfluss auf die Auswahl von alternativen Strukturen haben kann, bspw. Possessor-Angaben als Genitiv-NP oder PP im Englischen, oder die Position von Adverbien und anderen Konstituenten. Experimentelle Studien dazu belegen, dass bspw. das Adverb drin vorzugsweise in Positionen realisiert wird, wo es als nicht akzenttragende Silbe einen metrischen Typ vervollständigt. Entsprechend der Akzentposition im angrenzenden Substantiv wird das Adverb vor (17b) oder nach dem Nomen (16a) favorisiert, obwohl beide Varianten grammatikalisch korrekt sind.

(16a) Da wollte der Peter Tomaten drin kochen. (rhythmisch)

(16b) Da wollte der Peter Kohlrüben drin kochen. (nicht rhythmisch)

(17a) Da wollte der Peter drin Tomaten kochen. (nicht rhythmisch)

(17b) Da wollte der Peter drin Kohlrüben kochen. (rhythmisch)

(Vogel et al. 2015:271)

Analog zu (16) und (17) kann man annehmen, dass auch die Abfolgen von Adverbien und Partikeln wie in (15) sich nach dem vorliegenden Satzrhythmus ausrichten. In der Regel trägt das

12 HAZ09/JAN.00037 Hannoversche Allgemeine, 02.01.2009: 4 
Adverb eine höhere prosodische Prominenz als die Partikel und kann Akzentzusammenstöße mit seinen Nachbarn auslösen. Die Position der Partikel kann dem entgegenwirken und somit einen einheitlichen Rhythmus schaffen.

Um Effekte der Rhythmizität auf die Abfolge zwischen Adverb und schon/noch zu überprüfen, wurde eine experimentelle Untersuchung durchgeführt und Hypothese I getestet.

\section{HYPOTHESE I}

Die unrhythmischen Vorgaben werden beim Einsprechen der Abfolge der Partikel-Adverb-Sequenz zugunsten der rhythmisch ausgeglichenen Variante umgestellt. Die Oberflächenstruktur wird durch entsprechende prosodische Beschränkungen rhythmisch ausgewogen.

Da es zu erwarten ist, dass Probanden sich verstärkt an die schriftliche Vorgabe halten, sollte die Hypothese der rhythmischen Anpassung dann zutreffen, wenn mehr Abweichungen von der Vorgabe in den nicht-rhythmischen Target-Sätzen zu finden sind.

Neben der rhythmischen Anpassung ist es durchaus denkbar, dass Probanden die Umstellung der Sequenz aufgrund anderer Faktoren vornehmen. Ein möglicher Faktor ist die Verwendung unmarkierter und unmarkierter Abfolgen. Geht man davon aus, dass die funktionalen Köpfe einer Basisabfolge für Adverbien und Partikeln entsprechen, kann man zunächst annehmen, dass eine solche Abfolge als unmarkiert generiert wird und Variationen davon markierte Strukturen darstellen. Dieser Unterschied kann sich auf die Stellung der Partikel vor oder nach dem Adverb während der Produktion dahingehend auswirken, dass Sprecher unbewusst die Vorgabe verändern. Entsprechend lässt sich eine Hypothese II wie folgt formulieren:

\section{HYPOTHESE II}

Die Umstellung der Adverb-Partikel-Sequenz erfolgt zugunsten einer unmarkierten Grundabfolge.

Man kann erwarten, dass Probanden häufiger auf unmarkierte Sequenzen zurückgreifen, da sie in der Regel frequentierter gebraucht werden und einen breiteren Verwendungskontext haben. Sowohl für das Experiment als auch für einen Blick in Korpusdaten wurde auf gesprochene Sprache zurückgegriffen. Dies liegt insbesondere daran, dass sie einer höheren Variabilität unterliegen als geschriebene Texte. Unter der Annahme, dass bei geschriebener Sprache primäre Struktur-Generierungen durch redaktionelles Arbeiten stilistisch und normativ überarbeitet werden, sind gerade prosodische Einflussfaktoren primär in gesprochenen Daten zu finden.

\subsubsection{Experimentdesign und Durchführung}

Im Fokus der vorliegenden Untersuchung stehen variable Abfolgen der Partikeln schon und noch mit Adverbien, die einerseits eine Frequenz bzw. Habitualität ausdrücken (immer, oft) oder zukunftsbezogen sind (bald) und somit in der Regel einen imperfektiven Charakter haben. Andererseits werden die Partikeln auch mit Adverbien kombiniert, die je nach Kontext abgeschlossene oder andauernde Handlungen modifizieren (wie jetzt und heute). Dafür wurden 
sechs Adverb-Partikel-Sequenzen ${ }^{13}$ ausgewählt, deren Abfolge im Satz entweder rhythmisch angepasst oder umgestellt ist und einen rhythmischen Bruch verursacht.

Die Kombinationen aus Partikel und Adverb wurden in zwei unterschiedlichen rhythmischen Umgebungen untersucht. Zum einen in einer daktylischen Konfiguration, wo der Hauptakzent der Sequenz auf dem Adverb zu finden ist und die Partikel als zusätzliche unbetonte Silbe (cf. (18)) den Daktylus vervollständigt, sodass Abfolgen von einer akzenttragenden und zwei nicht akzentuierten Silben entstehen. Die Sequenzen in der daktylischen Umgebung wurden syntaktisch so eingebettet, dass sie die erste Position nach dem finiten Verb einnehmen. Die zweite rhythmische Umgebung stellten Sequenzen im Trochäus dar: auf eine betonte Silbe folgt eine unbetonte. Eine unbetonte Partikel kann hier deshalb entweder nach der akzentuierten Silbe des Adverbs den Fuß vervollständigen oder als „Puffer“ zwischen dem Adverb und einer vorangehenden akzenttragenden Silbe einen Akzentzusammenstoß verhindern.

(18a) Daktylus [+rhythmisch]

Beate trug immer schon schräge Klamotten, doch dieses Ensemble ist einfach nur Schrott.

(18b) Daktylus [-rhythmisch]

Beate trug schon immer schräge Klamotten, doch dieses Ensemble ist einfach nur Schrott.

(18c) Trochäus [+rhythmisch]

Der Winzer trinkt den Wein schon immer, den stellt er auch selber her.

(18d) Trochäus [-rhythmisch]

Der Winzer trinkt den Wein immer schon, den stellt er auch selber her.

Die syntaktische Einbettung der Adverb-Partikel-Sequenz erfolgt in der Trochäus-Umgebung am rechten Rand der Intonationsphrase (iP). Man kann davon ausgehen, dass an dieser Stelle neben der Rhythmizität weitere prosodische Anpassungen zu erwarten sind, die den Beschränkungen der FinALFocus-Familie unterliegen (cf. dazu Selkirk 1996, 2011). So ist es beispielsweise möglich, Partikeln oder andere Funktionswörter in letzter Position mit einem starken Akzent zu realisieren und bestimmte pragmatische Markierungen kenntlich zu machen.

Für beide Rhythmus-Umgebungen wurden Minimalpaare gebildet, die sich entweder in das vorgegebene Rhythmusmuster der Target-Sätze problemlos einfügen [+rh] (cf. 18a und 18c) oder gegen dieses Verstoßen [-rh] (18b und 18d). Für die sechs unterschiedlichen Sequenzen mit jeweils vier lexikalischen Targetsätzen konnten somit insgesamt 2x6x4 rhythmische und 2x6x4 nicht rhythmische Varianten gebildet werden. Um Redundanzen während der Testphase zu vermeiden, erfolgte die Verteilung der Stimuli nach dem Design des Lateinischen Quadrats auf vier Testversionen, sodass jeder Teilnehmer einen Targetsatz in einer [+rh] oder [-rh]-Variante als Stimulus (insgesamt 48) zu bearbeiten hatte. Hinzu kamen 30 Füllsätze, die keine strukturelle oder rhythmische Verbindung zu den Adverb-Partikel-Sequenzen hatten.

An der Studie nahmen insgesamt 26 (20f, 6m) Probanden gegen Bezahlung teil. Den Großteil machten dabei Studierende der Universität Bielefeld aus, die überwiegend aus dem norddeutschen Sprachraum kamen.

13 immer schon; oft schon; jetzt schon; bald schon; immer noch; heute noch. 
Die Aufnahmen erfolgten in einer schallisolierten Sprecherkabine. Dabei saßen die Teilnehmerinnen und Teilnehmer vor einem Bildschirm, wo „Interview-Ausschnitte“ in schriftlicher Form präsentiert wurden. Die Aufgabe bestand darin, sich die Antwort des Interviews (=Stimulus) einzuprägen und sie im Anschluss einzusprechen. Während der Aufnahme verschwand die Vorgabe, sodass das Einsprechen aus dem Gedächtnis heraus erfolgt ist.

Sowohl die Präsentation am Bildschirm als auch die Aufnahme erfolgten automatisch mittels DMDX (Forster und Foster 2003) bei einem Zeitintervall von 12000ms für die schriftliche Präsentation und 8000ms für das Einsprechen.

Insgesamt wurden 1248 Sätze eingesprochen, von denen 68 als ungültig ausgeschlossen werden, weil dort die Adverb-Partikel-Sequenz vollständig oder anteilig fehlt.

\subsubsection{Ergebnisse: Test der Rhythmizität}

Die Ergebnisse für Hypothese I sehen folgendermaßen aus: Aus 1248 Aufnahmen und davon 1180 gültigen Produktionen entsprechen erwartungsgemäß 89,32\% der vorgegebenen AdverbPartikel-Abfolge. In 126 Fällen (10,68\%) haben die Probanden die schriftliche Vorgabe verändert und die Abfolge beim Einsprechen umgestellt. Dabei kommt heraus, dass ein signifikanter Unterschied bezüglich der Umstellung nach Rhythmizität nur bei Sätzen im Daktylus besteht (siehe Tabelle 1). Das trochäische Muster bringt keine signifikanten Unterschiede zwischen rhythmisch angepasster und unangepasster Abfolge der betrachteten Elemente hervor.

Der T-Test einer unabhängigen Stichprobe für Daktylus $\left(\mathrm{n}_{+\mathrm{rh}}=299 ; \mathrm{n}_{\text {-rh }}=290\right)$ und Trochäus $\left(\mathrm{n}_{+\mathrm{rh}}=294 ; \mathrm{n}_{-\mathrm{rh}}=297\right)$ gibt an, dass sich im Daktylus beide Gruppen (+rh und -rh) signifikant voneinander unterscheiden und unter einer Bedingung häufiger Umstellungen vollzogen werden als unter der anderen.

\begin{tabular}{|l|l|c|c|c|}
\hline & & T & Df & Sig.(2-seitig) \\
\hline Daktylus & Varianzen sind gleich & 3,836 & 587 &, 000 \\
\hline & Varianzen sind nicht gleich & 3,853 & 546 &, 000 \\
\hline Trochäus & Varianzen sind gleich & 0,209 & 589 &, 835 \\
\hline & Varianzen sind nicht gleich & 0,209 & 588 &, 835 \\
\hline
\end{tabular}

Tabelle 1: T-Test für die Mittelwertgleichheit

Ein genauer Blick auf die Daten zeigt jedoch, dass nicht die unrhythmischen Vorgaben umgestellt werden. Entgegen der Erwartung fällt die Anzahl der Umstellungen sowohl im Daktylus als auch im Trochäus in rhythmisch angepassten Vorgaben höher aus (Tabelle 2).

\begin{tabular}{|c|c|c|c|}
\hline & & Entsprechend der Vorgabe & Umgestellt \\
\hline Daktylus & + rhythmisch & 235 & 64 \\
\hline & - rhythmisch & 261 & 29 \\
\hline Trochäus & + rhythmisch & 277 & 17 \\
\hline & - rhythmisch & 281 & 16 \\
\hline
\end{tabular}

Tabelle 2: Absolute Zahlen für die eingesprochenen Daten

Als erstes Zwischenfazit kann man somit festhalten, dass die Abfolge der Adverbien und der Partikeln zueinander auf der Oberflächenstruktur nicht aufgrund von rhythmischen Strukturen 
erfolgt, sondern anderen Parametern unterliegen muss. Die Hypothese I kann daher nicht bestätigt werden.

Die Auswertung der aufgenommenen Daten zeigt, dass Probanden bei der Reproduktion der Sätze aus dem Gedächtnis Umstellungen der schriftlichen Vorgabe vollziehen, die nicht auf den Rhythmus zurückzuführen sind. So werden im Daktylus aus insgesamt 93 umgestellten Fällen bestimmte Sequenzen häufiger umgestellt als andere (cf. Tabelle 3).

\begin{tabular}{|c|c|c|}
\hline & \multicolumn{2}{|c|}{ Anteil an Umstellungen in \% $(\mathrm{n}=$ Anzahl der gültigen Fälle $)$} \\
\hline Vorgabe & {$[+\mathrm{rh}]$} & {$[-\mathrm{rh}]$} \\
\hline immer schon & $81,8(22)$ & $12,2(4)$ \\
\hline immer noch & $33,3(6)$ & $66,7(12)$ \\
\hline heute noch & $22,2(2)$ & $77,8(7)$ \\
\hline jetzt schon & $33,3(2)$ & $66,7(4)$ \\
\hline bald schon & $100(19)$ & 0 \\
\hline oft schon & $89,5(17)$ & $10,5(2)$ \\
\hline
\end{tabular}

Tabelle 3: Anzahl umgestellter Paare entsprechend der rhythmischen Anpassung im Daktylus

Die Tabelle 3 ist so zu lesen, dass die Abfolge unter „Vorgabe“ der rhythmisch angepassten Einbettung in den Satz entspricht (z. B. immer schon). Die [-rh]ythmische Variante ist dabei das umgestellte Minimalpaar (schon immer) mit dem identischen Trägersatz und somit gleichem Grundrhythmus; lediglich das Adverb und die Partikel sind umgestellt.

Aus den Daten kann man ablesen, dass die $\mathrm{Adv}+$ schon-Sequenzen (immer schon, bald schon, oft schon) überwiegend (> 80\%) in einer rhythmischen Umgebung umgestellt werden. Die unrhythmische schon-Adv-Sequenz wird jedoch nur im Fall von jetzt schon verändert. Anders formuliert: Es herrscht eine starke Präferenz für die Strukturen schon immer, schon bald, schon oft und jetzt schon. Man stellt also fest, dass die Probanden rhythmisch disharmonische Strukturen in Kauf nehmen und [schon Adverb] auf der Oberfläche favorisieren.

Für den Fall der Adv-noch-Sequenz sieht es so aus, dass die nicht-rhythmischen Varianten zu einem nicht unerheblichen Anteil (66,7\% noch immer und 77,8\% noch heute) zugunsten der nachgestellten Partikel verändert werden. Die Daten legen daher nahe, dass im gegebenen Experiment eine starke Präferenz für die Position von schon vor dem Adverb erfolgt, wogegen noch tendenziell nach dem Adverb favorisiert wird. Dieser Unterschied zwischen [Adv noch] und [schon Adv] lässt sich statistisch ermitteln: Mittelwertvergleiche zwischen den Paaren (ANOVA: $\mathrm{F}=11,656 ; \mathrm{df}=5 ; \mathrm{p}<.000)$ zeigen, dass sich zwei homogene Gruppen ergeben (TukeyTest bei angenommener Varianzgleichheit). Zum einen die [Adv noch]-Gruppe mit der Präferenz zu einer nachgestellten Partikel, zu der zusätzlich jetzt schon gehört; und zum anderen die übrigen [schon Adv]-Paare mit der Tendenz zu einer vorangestellten Partikel.

Ähnliche Tendenzen lassen sich auch aus den Daten der trochäisch eingebundenen Sequenzen ablesen (Tabelle 4): Die rhythmisch angepasste Vorgabe von oft schon wird überwiegend im Output zu schon oft verändert. Allerdings ist die Anzahl der Fälle von Umstellung in dieser Bedingung mit $\mathrm{n}=33 \mathrm{zu}$ gering, um hier verlässliche statistische Vergleiche durchführen zu können. 


\begin{tabular}{|c|c|c|}
\hline & \multicolumn{2}{|c|}{ Anzahl umgestellter Fälle } \\
\hline Vorgabe & {$[+\mathrm{rh}]$} & {$[-\mathrm{rh}]$} \\
\hline schon immer & 0 & 2 \\
\hline noch immer & 3 & 1 \\
\hline noch heute & 2 & 5 \\
\hline jetzt schon & 1 & 5 \\
\hline bald schon & 0 & 1 \\
\hline oft schon & 11 & 2 \\
\hline
\end{tabular}

Tabelle 4: Anzahl umgestellter Paare entsprechend der rhythmischen Anpassung im Trochäus

Auch wenn beide Sequenzen [Adv Part] und [Part Adv] auf der Oberfläche gleichermaßen akzeptabel sind und keine wesentlichen Unterschiede in der Interpretation bzw. Skopusresolution aufweisen (cf. Abschnitt 2 und Abschnitt 3), gibt es auf Seiten der Sprecher Präferenzen für die eine oder die andere Sequenz. So wird schon vorwiegend vor dem Adverb realisiert, während noch dem Adverb folgt. Die Präferenz für die eine oder die andere Abfolge hängt also stärker von der lexikalischen Form der Partikel ab als von der Funktion der Partikeln als Aspektmodifikatoren.

\subsubsection{Ein Blick in Daten von gesprochenen Korpora}

Dass die Sprecher des Deutschen eine Präferenz zur Voranstellung von schon und zum Nachstellen von noch auch außerhalb der Experimentdaten haben, lässt sich an der Auswertung der Verwendungszahlen in den Korpora der gesprochenen Sprache (Datenbank für gesprochenes Deutsch 2) ablesen. Die absoluten Zahlen in Tabelle 5 geben die Summe der Vorkommen einer Sequenz x sowie ihre umgestellten Gegenpaare in spontanen Gesprächen unterschiedlicher Korpora $^{14}$.

\begin{tabular}{|c|c|c|}
\hline $\mathrm{x}$ & $\begin{array}{c}\text { Anzahl der Treffer für } \\
\text { Sequenz } \mathrm{x}\end{array}$ & $\begin{array}{c}\text { Anzahl der Treffer für } \\
\text { Umstellung von } \mathrm{x}\end{array}$ \\
\hline immer noch & 642 & 66 \\
\hline heute noch & 216 & 24 \\
\hline jetzt schon & 190 & 15 \\
\hline oft schon & 11 & 31 \\
\hline immer schon & 36 & 120 \\
\hline schon bald & 9 & 1 \\
\hline
\end{tabular}

Tabelle 5: Vorkommen der Sequenz in DGD2

Die erste Beobachtung ist, dass beide Abfolgen für Adverbien und Partikeln in gesprochenen Daten zu finden sind. Also wird keine der lexikalischen Kombinationen zwischen Adverb und schon/noch im realen Sprachgebrauch ausgeschlossen. Die im Experiment beobachtete Systematik bildet sich auch hier ab: Die beiden [Adverb noch]-Paare werden mit Abstand häufiger verwendet als die [noch Adverb]-Sequenzen (642:66 für immer noch vs. noch immer; 216:24 für heute noch vs. noch heute). Auch die Präferenz für jetzt schon ist deutlich höher als für

14 Ausgeschlossen wurden hierbei spezifische Mundartenkorpora oder Gespräche im Ausland gebrauchter Varietäten. Die Dauer der für die Korpusauswertung verwendeten Gespräche beträgt insgesamt 345:07 Stunden. 
schon jetzt (190:15). Im Fall von oft zeichnet sich eine leichte Präferenz zu einem vorangestellten schon ab. Diese wird im Fall von immer mit 36 zu 120 Treffern noch stärker. Lediglich die Kombination von schon und bald lässt keine Aufschlüsse zu einer eindeutigen Präferenz zu. In den betrachteten Korpora kommt sie viel zu selten vor, um hier eindeutige Aussagen zu treffen bzw. eine zufällige Verteilung auszuschließen.

Somit kann man festhalten, dass sowohl aus den Daten des Experiments als auch dem Korpus eine Grundabfolge ablesbar ist und die Hypothese II zum Teil bestätigt werden kann. Allerdings handelt es sich hier nicht um eine funktionalbasierte Ordnung, bei der man generell sagen kann, dass die Partikel vor oder nach einem Temporaladverb steht. Vielmehr handelt es sich um eine lexikalisch basierte Abfolge, die eine Trennung zwischen schon und noch abzeichnet, jedoch nicht für alle Adverbien gleichermaßen gilt.

\subsubsection{Prosodische Auffälligkeiten der Daten}

Als einen dritten und letzten prosodischen Faktor, der hier als relevant für die Wortstellung ausfällt, kann man die vordefinierte Position des Primärakzents innerhalb von Intonationsphrasen oder kleineren prosodischen Einheiten betrachten. So führt bspw. Zubizarreta (1998) Umstrukturierungen im linearen Output in Sprachen wie Spanisch und Italienisch auf den Prozess des P(rosodic)-Movements zurück. Aus der Vorannahme, dass der Fokus und somit der Primärakzent bei einer neutralen Akzentuierung entsprechend der NUCLEAR STRESS RULE (Chomsky/Halle 1968) satzfinal realisiert wird, tragen im Spanischen in der Regel das Objekt oder das intransitive Verb den Primärakzent. Subjekte, die als prototypische Vertreter der bereits bekannten Information und daher [-focus]-markiert sind, werden aus einer solchen akzenttragenden und satzfinalen Position herausbewegt. Das Resultat dieser Analyse ist, dass die SVO-Struktur in diesen Sprachen eine Oberflächenabfolge ist, die sich an den Akzentuierungen, insbesondere an der Fokusmarkierung, ausrichtet und aus der VOS-Struktur abgeleitet wird. Ähnliche Umstellungseffekte der Fokusmarkierung lassen sich auch im Deutschen beobachten, bspw. an der Abfolge der Objekte im Mittelfeld (cf. Büring 1999, 2000). Eine unmarkierte Standardabfolge Dativobjekt > Akkusativobjekt kann auf der Oberfläche verändert werden, wenn das Dativobjekt im engen Fokus steht. Somit erhält es den Primärakzent und wird der Forderung nach einem solchen Akzent in phrasenfinalen Positionen gerecht (FINAL FocUS-Beschränkung in Büring 1999: 5). ${ }^{15}$

Ähnliche prosodische Effekte können auch hinter der Adverb-schon/noch-Variation vermutet werden. Ausgehend davon, dass innerhalb der Akzentdomäne das Adverb und nicht die Partikel den Hauptakzent trägt, kann man annehmen, dass ein nachgestelltes Adverb dann favorisiert wird, wenn es prosodisch salient (bspw. durch eine emphatische oder eine enge Fokussierung) ist.

\footnotetext{
15 Tatsächlich ist es so, dass Scrambling und Akzentuierung im Deutschen wesentlich komplexer ausfallen, als dargestellt. Auf solche komplexen Fälle wird hier verzichtet, da sie nicht vom besonderen Interesse für die Arbeit sind. Lediglich die Idee, dass eine satzfinale Position eine besondere prosodische Markierungsposition ist, soll hier als möglicher Faktor für die Akzentuierung und Umstellung der Adverb-Partikel-Sequenz übernommen werden. Deshalb wird in der vorliegenden Analyse auf einfache syntaktische Strukturen, wie in Keenan/Stabler (2003) vorgeschlagen, zurückgegriffen.
} 
Wertet man die Daten des Experiments unter dem Aspekt der hervorgehobenen Akzentuierung ${ }^{16}$ der Adverbien aus, lässt sich Folgendes beobachten: Keine eingesprochenen Stimuli im Daktylus zeigen eine prosodische Hervorhebung am Adverb. Bei diesen Stimuli ist die AdverbPartikel-Sequenz unmittelbar nach der V2-Position eingebettet. Der „FINAL-Focus-Effekt" hat hier keine Auswirkungen auf die Akzentuierung und die Umstellung. Anders sieht es jedoch in den Stimuli der Trochäus-Bedingung aus. Hier wurde die Sequenz in satzfinaler Position eingebettet und zeigt Unterschiede in der Akzentuierung der Adverbien in Korrelation zu ihrer Abfolge mit der Partikel $\left(\mathrm{r}_{\mathrm{s}}=.341, \mathrm{p}<.000\right)$. Insbesondere in den unrhythmischen Stimuli mit einer [Adverb Partikel]-Abfolge tragen die Adverbien einen perzeptiv stärker wahrnehmbaren Primärakzent (siehe hellgrüner Balken in Abbildung 2) und intendieren eine kontrastive Lesart. ${ }^{17}$

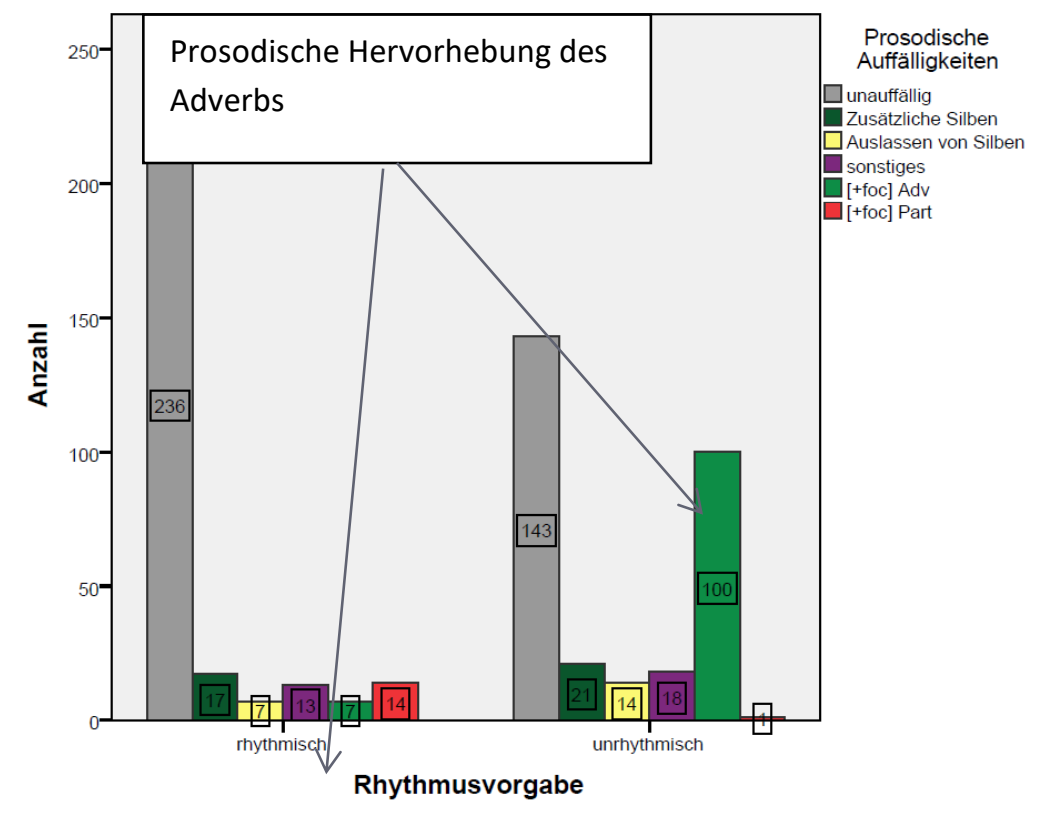

Abbildung 2: Prosodische Auffälligkeiten bei der Realisierung von rhythmischen
und nicht rhythmischen Trochäen ${ }^{18}$

Entgegen der Erwartung ist es nicht das Adverb in letzter Position, das eine prosodische Markierung trägt, sondern das Adverb, worauf die Partikel folgt. Zwei mögliche Erklärungen sind für die Beobachtung denkbar: a) es ist der rhythmische Bruch innerhalb der Intonationsphrase,

\footnotetext{
${ }^{16}$ Als Anzeichen für hervorgehobene Akzentuierung wird hier auf die perzeptive Wahrnehmung einer erhöhten Prominenz der Akzenttragenden Silbe am Adverb oder ggf. der Partikel zurückgegriffen. I. e. die betroffene Silbe zeichnet sich durch deutlich wahrnehmbare längere Dauer und einen Anstieg im Energieniveau aus. Inwiefern es sich bei den Aufnahmen um einen engen oder einen kontrastiven Fokus handelt, oder ein emphatischer Nachdruck verliehen wird, kann nicht gesagt werden.

${ }^{17}$ Die nicht rhythmischen Aufzeichnungen zeichnen sich vermehrt durch weitere prosodische Auffälligkeiten wie auslassen oder hinzufügen von Silben oder Akzentverschiebung aus. Da sie nicht im Mittelpunkt der vorliegenden Analyse stehen, werden sie hier erwähnt, jedoch nicht weiter ausgeführt werden.

18 Die Anzahl der unauffälligen prosodischen Realisierungen ist in rhythmisch ausgewogenen Stimuli höher als in denen, wo die Adverb-Partikel-Sequenz unrhythmisch ist. Die Unrhythmizität wird jedoch nicht immer mittels Umstellung der beiden Einheiten „repariert“ sondern unteranderem dadurch, dass Probanden Silben reduzieren oder auslassen bzw. zusätzliche Silben (einsilbige Partikeln wie ja, halt, doch) hinzufügen. Besonders fällt jedoch der signifikante Anstieg der fokussierten Adverbien in der [-rh]-Bedingung auf: Nur sieben Fälle betrifft es in der [+rh]-Bedingung, jedoch 100 in der anderen (T-Test bei einer Stichprobe mit $\mathrm{T}=80,552, \mathrm{df}=106, \mathrm{p}>.000$ ).
} 
der eine besondere Aufmerksamkeit nach sich zieht und deshalb das Adverb als salient herausstellt; oder b) das optimale Akzentmuster der fokussierten prosodischen Wörter ist trochäisch (cf. Wiese 1996). Dadurch, dass die Partikel in die Akzentdomäne des Adverbs integriert wird, entsteht hier eine optimale akzenttragende Fuß-Struktur (Adverb Partikel $_{\mathrm{W}}$ ) $_{\mathrm{AD} / \mathrm{Fu}}$ mit einem trochäischen Akzentmuster. Womöglich stellen solche Strukturen bessere Fokus-Exponenten dar als ihre umgestellten Varianten. Welche Erklärung tatsächlich plausibel ist, lässt sich an den vorliegenden Daten leider nicht eindeutig klären und bedarf weiterer Untersuchungen.

Zusammenfassend kann man sagen, dass die Annahme von ausgewiesenen Akzentpositionen wie die FINAL FOKUS-Beschränkung eine Auswirkung auf die Adverb-Partikel-Abfolge haben kann; allerdings nur, wenn die Sequenz auch in entsprechenden Positionen zu finden ist. Das bedeutet, dass Sprecher bspw. in satzfinalen Positionen gezielt [Adverb Partikel] für einen starken Primärakzent auf dem Adverb verwenden und es kontextuell kontrastieren, wogegen [Partikel Adverb] nicht immer eine solche prosodisch hervorgehobene Markierung aufweist. Die Varianz außerhalb solcher ausgewiesenen Positionen ist jedoch nicht auf prosodische Rhythmus- oder Akzentstrukturen zurückführbar.

\section{Diskussion der Daten}

Fasst man die Faktoren zusammen, die die Abfolge der Temporaladverbien und der Partikeln schon/noch bestimmen, ergibt sich soweit das Bild, dass aus der prosodischen Sicht weder Heaviness-Effekte noch die rhythmische Anpassung ausschlaggebend sind. Dies liegt insbesondere daran, dass neben der Umstellung weitere Prozesse vorgenommen werden können, die ebenfalls zur Rhythmizität beitragen (gemeint sind insbesondere Reduktionseffekte bis hin zur Tilgung von Silben oder anderweitige Manipulation der Silbendauer). Aus der syntaktischen Perspektive heraus gibt es auch keine eindeutige Restriktion: sowohl die Abfolge [Partikel Adverb] als auch [Adverb Partikel] sind auf der Oberfläche zulässig. Schnittstelleneffekte zwischen prosodischen und syntaktischen Beschränkungen lassen sich an entsprechenden Stellen wie der satzfinalen Position beobachten; sie sind jedoch nicht durchgängig für alle Fälle relevant.

Semantisch betrachtet ergeben beide Abfolgen gleiche Resultate in Bezug auf die Propositionen der ausgedrückten Ereignisse, sodass man auch an dieser Stelle nicht von einer distinktiven Verteilung und somit unterschiedlichen Interpretationen sprechen kann. Dennoch erkennt man sowohl in den Korpora als auch in den Daten des Experiments eindeutige Präferenzen für die Abfolgen [schon Adverb] und [Adverb noch]. Dies ist insofern ungewöhnlich, als dass beide Partikeln funktional zur gleichen Kategorie zugeordnet werden, sei es in der Angabe zum perfektiven/imperfektiven Aspekt einer Handlung oder in der Funktion der Angabe zur zeitlichen Abfolge von Phasen eines Ereignisses bzw. den Erwartungen des Sprechers zu der Phasenabfolge (cf. Abschnitt 2). Eine kategoriale Generalisierung innerhalb der Grammatik sollte daher darauf hinauslaufen, dass die Position zum Adverb weniger funktional, sondern viel mehr lexikalisch festgelegt wird.

Es kommen noch zwei weitere Probleme hinzu, die eine kategoriale Grammatikregel erschweren: a) man kann nicht alle Adverbien, die temporale Modifikation auslösen, gleich betrachten. Bereits in den hier untersuchten Fällen unterscheiden sich frequenzangebende Adverbien (oft) von denen, die Zeitpunkte (jetzt) oder Dauer (heute) beschreiben (siehe Abschnitt 3). Nehme 
man mehr als die hier sechs besprochenen Adverbien, wären wahrscheinlich noch weitere Unterschiede zu erwarten, bspw. bei habituellen Angaben. Und wie in Abschnitt 1 angesprochen, sollte sowohl die semantische Klasse der Verben als auch die kontextuelle Einbettung der Sätze in die Analyse einbezogen werden, weil auch sie zu Ausnahmen in Generalisierungen führen können.

Neben den Adverbien sind b) auch die beiden Partikeln nicht fest in ihrer Position. Man kann annehmen, dass die beiden Typen sich dahingehend unterscheiden, als dass schon vor dem Adverb realisiert wird und noch danach, ${ }^{19}$ Auf der Token-Seite äußert sich dennoch eine starke Präferenz für jetzt schon, also einem nachgestelltem schon (cf. Abschnitt 4.2.2). Die lexikalisch festgelegte Kombination der Abfolgen von schon und noch mit Adverbien ist also auch hier nicht eindeutig generalisierbar.

Man kann an dieser Stelle festhalten, dass es nicht möglich ist, entweder aus der semantischen, der syntaktischen oder der prosodischen Komponente heraus, eindeutige grammatische Regeln $\mathrm{zu}$ formulieren, die die Abfolgen der Adverbien und der Partikeln zueinander auf der Oberfläche kategorisch regeln. Eine Möglichkeit mit dieser Beobachtung umzugehen ist, für solche Fälle eine absolut freie Variation anzunehmen: Die Grammatik des Deutschen lässt beide Abfolgen völlig gleichberechtigt zu. Gegen eine solche Pauschalisierung spricht allerdings einerseits die Intuition, dass die Minimalpaare sich doch in einer subtilen Weise voneinander unterscheiden und nicht völlig identisch sind. Andererseits lassen sich erwähnten Präferenzen bei den Abfolgen festlegen, auch wenn sie nicht immer spezifischen lexikalischen oder funktionalen Kategorien entsprechen.

Die andere Möglichkeit, ein Grammatikkonzept für diese Fälle zu erstellen, besteht in der Annahme von Modellen, die sich mit frequentativem Gebrauch von Strukturen auseinandersetzen wie bspw. die Exemplar-Theorie (cf. Bybee 2006, Pierrehumbert 2006, Guy 2014 und darin enthaltene Verweise) oder kollokations- und kookkurrenzstatistische Modelle (cf. Evert 2008, Herbst 1996, Stefanowitsch/Grice 2003). Solche frequenzbasierte oder probabilistische Modelle nehmen die Häufigkeiten der Strukturen in der natürlichen Sprachverarbeitung als ein psycholinguistisches Phänomen und leiten draus gradiente Daten der grammatikalischen Struktur ab. Autoren wie Jurafsky (2003) oder Manning (2003) sprechen sich dafür aus, die grammatischen Strukturen nicht immer regelbasiert abzuleiten. So argumentieren die Autoren dafür,

\footnotetext{
${ }^{19}$ Spekuliert man über die Gründe, warum ein schon vorangestellt und ein noch nach dem Adverb erscheint, kann man bspw. die Funktion von schon als Modalpartikel salienter betrachten als die von noch. Insbesondere in nachgestellter Position ist schon bei konzessiven Kontexten betonbar (i a), ein emphatisch hervorgehobenes noch ist hier nicht möglich.

(i)a Ich brauche die Daten heute schón, aber eigentlich komme ich auch ohne aus.

(i)b Ich brauche die Daten heute *nóch, aber eigentlich komme ich auch ohne aus.

Tatsächlich gab es einige wenige Fälle in der Produktionsstudie, in denen Probanden in der satzfinalen Position der Sequenz bei entsprechend möglichen Kontexten die Abfolge [schon Adverb] mit dem Hauptakzent auf dem Adverb eingesprochen haben, die gleiche Paarung in der Abfolge [Adverb schon] jedoch mit einem Akzentton auf schon und somit als Modal- und nicht mehr als Aspektpartikel realisiert haben. Eine solche Akzentverschiebung ist in den noch-Stimuli nicht vorgekommen. Man kann an dieser Stelle spekulieren, ob die beiden Positionen für schon als desambiguierend zu verstehen sind: [schon Adverb] für die Aspekt- und [Adverb schon] für die Modalpartikel - also ein Koerzionseffekt, der sich auch in der linearen Abfolge äußert. Und weil noch keine solche Verteilung hat, äußert es sich in beiden Positionen als Aspekt-Partikel.
} 
dass Sprecher durchaus in der Lage sind, nicht nur lexikalische Einheiten im Gedächtnis abzuspeichern, sondern auch höher frequentierte Kollokationen sowie längere Phrasen und Sätze. Der stetige Input aus der Sprachumgebung führt dazu, dass der Bestand der abgespeicherten Strukturen kontinuierlich und inkrementell aktualisiert und auf den neuesten Stand gebracht wird. In empirischen Betrachtungen des alltäglichen Sprachgebrauchs findet man nicht selten widersprüchliche Beschränkungen und vermeintliche Regelverletzungen auf unterschiedlichen Ebenen (Phonologie, Morphologie, Syntax), die eher auf Konventionen und weniger auf kategoriale Regelbildung hindeuten. Manning (2003:296) schreibt dazu: ,[...] generative linguistics has produced many explanatory hypotheses of considerable depth, but is increasingly failing because its hypotheses are disconnected from verifiable linguistic data.“

Ebendiese Problematik lässt sich auf die Abfolge der Temporaladverbien und der Partikeln übertragen: Es reicht nicht aus, die beiden funktionalen Kategorien zu einander zu ordnen, da sowohl die Klasse des Adverbs (Zeitpunkt vs. Frequenz; jetzt vs. heute etc., cf. Abschnitt 2) näher klassifiziert werden muss, als auch die Partikeln schon und noch verschiedene Präferenzen zeigen (siehe Abschnitt 4.2). Man kann daher nicht pauschal behaupten, dass die unmarkierte Position von schon vor einem Temporaladverb definiert ist. Sobald schon in Verbindung mit jetzt steht, ist die unmarkiete Abfolge [jetzt schon] und nicht umgekehrt. Ebensolche häufigen Verwendungen im Sprachgebrauch führen dazu, dass Sprecher bestimmte Strukturen als „chunks“ abspeichern (siehe Bybee 2010:33f.). Chunking als Prozess stellt die kognitive Basis zur Ausbildung von morpho-syntaktischen Konstruktionen bereit und wird aus der Verwendungshäufigkeit der entsprechenden Konstruktion im Sprachgebrauch untermauert. Bybee schreibt dazu:

"All instances of this construction that have been experienced by a language user have had some impact on the representation of the construction, though they may not all be lodged in memory. As with other memories, non-reinforced exemplars may become inaccessible or forgotten; both recency and frequency play a role in the maintenance of particular exemplars of constructions."

(Bybee 2010:28)

Für die Oberflächenabfolge von Temporaladverbien und die Partikeln schon/noch im deutschen Mittelfeld kann man festhalten, dass die Abfolge nicht aufgrund der funktionalen Ähnlichkeit von schon und noch erfolgt. Auch wenn beide Partikeln überwiegend die Aufgabe haben, Aussagen bezogen auf die (Im-)Perfektivität bzw. Phasenabfolgen zu modifizieren (siehe Abschnitt 2), werden sie innerhalb der Konstruktion mit einem Adverb unterschiedlich positioniert. Ein mögliches Exemplar kann wie in (19) aussehen: Sowohl schon als auch noch haben ihre eigenen funktionalen und sprachgebrauchsbezogenen Merkmale, die im Lexikon abgespeichert werden. $\mathrm{Zu}$ diesen Merkmalen gehören alle die, die die beiden Partikeln als Schnittmenge für die Aussagenmodifikation tragen, aber auch spezifische Merkmale, die kontextuell abgeleitet werden wie additive, modale, iterative etc. Lesarten (cf. schon ${ }_{1 / 2}$ und noch ${ }_{1 / 2}$ in Hoepelman/Rohrer (1981)). Unterschiede ergeben sich in der Position zum Adverb, wo sie empirisch nachweisbar unterschiedlich Präferiert werden (siehe Abschnitt 4.2). 
(19)

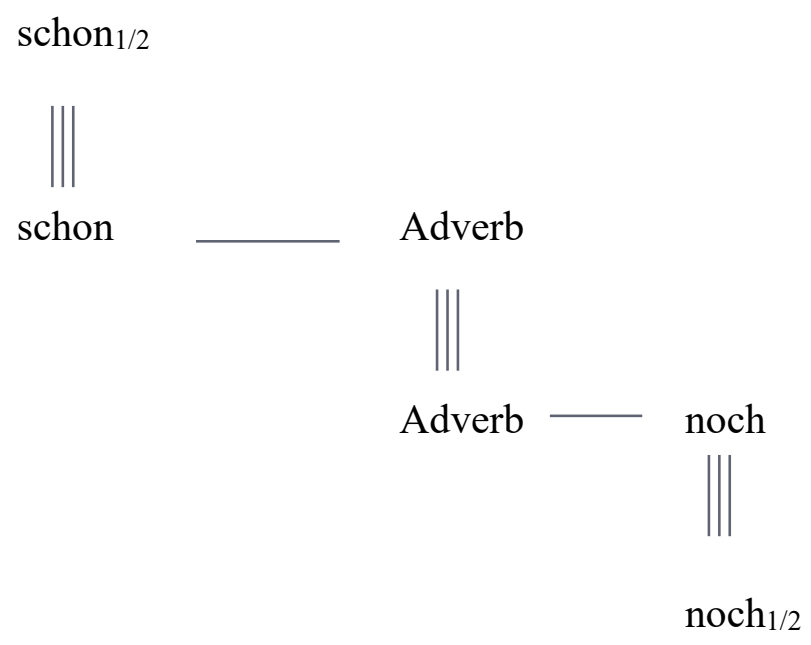

Eine Modellierung wie in (19) umgeht zwar die Problematik der Regelbildung anhand von funktionalen Gemeinsamkeiten beider Partikeln, erklärt jedoch noch nicht die Spezialfälle wie [jetzt schon]. Aus der Sicht der probabilistisch-psychologischen Sprachverwendung handelt es sich dabei um einen „Chunk“, der als solcher abgespeichert wird.

Für beide Partikeln gilt, dass sie sowohl vor als auch nach dem Adverb nicht ausgeschlossen sind; informationsstrukturelle oder stilistische Gründe können sich auf die Output-Variante auswirken und die unmarkierte Abfolge verändern. Geschieht dies im Sprachgebrauch so häufig, dass sie markierte Abfolge frequentierter anzutreffen ist als die unmarkierte, kann sie als Chunk abgespeichert werden, so auch in Fällen wie [jetzt schon]. Ein kurzer Blick auf die Daten in der DGD $^{20}$ bestätigt, dass die Wahrscheinlichkeit schon vor dem Temporaladverb anzutreffen höher ist als für ein nachgestelltes schon in Verbindung mit demselben Adverb (Tab. 6). Lediglich die Sequenz jetzt schon unterscheidet sich deutlich davon. ${ }^{21} 22$

\footnotetext{
${ }^{20}$ Ausgeschlossen wurden hierbei alle Mundartenkorpora und Korpora zur deutschen Sprache im Ausland, weil hier von der deutschen Umgangssprache abweichende Beschränkungen möglich sind. Relevante Korpora sind Dialogstrukturen (DS), Elizitierte Konfliktgespräche (EK), Forschungs- u. Lehrkorpus für gesprochenes Deutsch (FOLK), Deutsche Hochlautung (HL), Deutsche Standardsprache, König-Korpus (KN) und Pfeffer-Korpus (PF).

${ }^{21}$ Die Häufigkeitsverteilung der wahrscheinlichen Nachbarschaft der Adverbien und noch ist bei heute noch nicht so ausgeprägt, wie in den absoluten Zahlen des Korpus (Tab. 5). Dies liegt u. a. daran, dass für die Ermittlung der Treffer für noch (Gleiches gilt für schon) die syntaktische Einbettung oder die unterschiedlichen Lesarten nicht berücksichtigt wurden.

${ }^{22}$ Die Berechnung der Wahrscheinlichkeit für das Vorkommen der Partikel in Abhängigkeit der Adverb-Token erfolgt nach der Formel $p(w i \mid w i-1)=\frac{N(w i-1 w i)}{N(w i-1)}$

mit: N( $\left.w_{i}\right)$ als vom Vorgänger abhängiges Target der Sequenz, N( $\left.w_{i-1} w_{i}\right)$ als Anzahl der Treffer für die Sequenz beider Wörter, und N( $\left.\mathrm{w}_{\mathrm{i}-1}\right)$ als absolute Anzahl der Treffer für das erste Wort der Sequenz im Korpus (cf. Saffran, Aslin/Newport 1996).
} 


\begin{tabular}{|c|c|c|}
\hline & $\mathrm{p}$ für, schon $>\mathrm{Adv}^{“}$ & $\mathrm{p}$, Adv $>$ schon \\
\hline Bald & .052 & .0001 \\
\hline Jetzt & .0008 & .013 \\
\hline Oft & .027 & .0001 \\
\hline Immer & .014 & .003 \\
\hline immer & $\mathrm{p}$ für, ,noch $>\mathrm{Adv}^{\text {“ }}$ & $\mathrm{p} \mathrm{für}, \mathrm{Adv}>$ noch $^{\text {“ }}$ \\
\hline heute & .008 & .035 \\
\hline
\end{tabular}

Tabelle 6: Errechnete Wahrscheinlichkeiten für das Vorkommen der Adverbien in Verbindung mit schon/noch als direkte Nachbarn

Übertragen auf den Sprachgebrauchsprozess bedeutet es, dass ein Sprecher die Sequenzen, die er häufiger in seiner Sprachumgebung findet, entsprechend häufiger als Output produziert. Abweichende Abfolgen werden dabei als markierte Sequenzen mit einer neuen oder zusätzlichen Funktion innerhalb der Kontexte analysiert. Beispielhaft dafür sind die Daten des Experiments, wo die Abfolge [Adv Part] in satzfinaler Position mit einer verstärkten Akzentuierung des Adverbs einhergeht. Eine solche Form-Funktion-Beziehung kann allerdings auch dazu führen, dass die Erwartung an eine „Re-Interpretation“ gestellt wird, obwohl sie eigentlich überhaupt nicht gegeben ist. Man meint intuitiv eine andere Bedeutung zu haben; worin der Unterschied jedoch genau besteht, ist sowohl formal-semantisch als auch morpho-syntaktisch schwer zu beschreiben.

Zusammenfassend kann man also sagen, dass die konzeptuelle Grammatikvorstellung, die kategoriale Regeln sowohl für die Oberflächenstrukturen als auch ihre Interpretationen vorgibt, im Fall der hier betrachteten Partikeln und Adverbien sowohl zu viel als auch zu wenig aussagt: $\mathrm{Zu}$ viel, weil festgelegte Abfolgen keine Variation zulassen und keine brauchbaren Trigger für die Umstellung formulieren. Zu wenig, weil bei fehlenden Beschränkungen bestimmte empirisch belegbare Präferenzen und Ausprägungen für Strukturen unzureichend erfasst werden und verloren gehen. Auf den Sprachgebrauch ausgerichtete Modelle stellen für den Fall der hier beobachteten Variation ein geeigneteres Werkzeug bereit, die sowohl die Lernbarkeit der Struktur aus ihrer Häufigkeit ableiten und modifizieren können, als auch die kontextbedingten Verwendungen von funktional ähnlichen Strukturen mit ihren spezifischen Eigenschaften beschreiben.

\section{Literaturverzeichnis}

Andrews, Avery I. I. I. (1983): “A note on the constituent structure of modifiers". Linguistic Inquiry: 695-697.

Baker, Mark (1985): “The Mirror Principle and Morphosyntactic Explanation”. Linguistic Inquiry 16: 373-416.

Baker, Mark (1988): Incorporation: A theory of grammatical function changing. Chicago, IL: University of Chicago Press.

Behaghel, Otto (1932): Deutsche Syntax. Bd. 4. Eine geschichtliche Darstellung. Heidelberg: Carl Winters Universitätsbuchhandlung. (= Germanische Bibliothek).

Blumenfeld, Lev (2011): “Coercion and minimality”. The Linguistic Review 28(2): 207-240. doi:10.1515/tlir.2011.006 
Büring, Daniel (1999/2001): "Let's Phrase It! Focus, Word Order, and Prosodic Phrasing in German Double Object Constructions". In: Müller, Gereon/Sternefeld, Wolfgang (eds.): Competition in Syntax. Berlin/New York, Mouton de Gruyter: 69-105. (= Studies in Generative Grammar (StGG) 49).

Büring, Daniel (2000): What Do Definites Do That Indefinites Definitely Don't?. www.sfs.unituebingen.de/ astechow/Festschrift/Buring-5-komplett\%20fertig.pdf [06.11.2019]

Büring, Daniel (2003): “On D-trees, beans, and B-accents”. Linguistics and philosophy 26/5: 511-545.

Bybee, Joan L. (2006): “From Usage to Grammar: The Mind's Response to Repetition". Language 82/4: 711-733.

Bybee, Joan L. (2010): Language, usage and cognition. Cambridge etc.: Cambridge Univ. Press.

Choi, Hye-Won (1996): Optimizing structure in context. PhD thesis. Stanford, CA: Stanford University.

Chomsky, Noam/Halle, Morris (1968): The Sound Pattern of English. New York: Harper \& Row.

Cinque, Giuliano (1999): Adverbs and functional heads: A cross-linguistic perspective. Oxford: Oxford University Press. (= Oxford Studies in Comparative Syntax).

Cinque, Giuliano (2004): "Issues in adverbial syntax". Lingua 114: 683-710.

Cinque, Giuliano (2014): "Again on Tense, Aspect, Mood Morpheme Order and the 'Mirror Principle"”. In: Svenonius, Peter (ed.): Functional Structure from Top to Toe. The Cartography of Syntactic Structures 9. Oxford/New York, Oxford University Press: 232-265.

Comrie, Bernard (1976): Aspect. Cambridge etc. Cambridge University Press. (= Cambridge textbooks in linguistics 2).

Dik, Simon C. (1978): Functional grammar. Amsterdam: North-Holland. (= North-Holland linguistic series, 37).

Doherty, Monika (1973): “'Noch' and 'schon' and their Presupposition”. In: Kiefer, Ferenc/Ruwet, Nicolas (eds.): Generative Grammar in Europe. Dordrecht, Reidel: 154-177.

Ernst, Thomas (2002): The syntax of adjuncts. Cambridge: Cambridge University Press. (= Cambridge studies in linguistics 96).

Evert, Stefan (2008): “Corpora and collocations”. In: Lüdeling, Anke/Kytö, Merja (eds.): Corpus linguistics. An international handbook of the science of language and society. Berlin/New York, Mouton de Gruyter: 1212-1248.

Fanselow, Gisbert (1990): "Scrambling as NP-movement”. In: Grewendorf, Günther/Sternefeld, Wolfgang (eds.): Scrambling and Barriers. Amsterdam/Philadelphia, Benjamins: $113-142$.

Farkas, Donka. F./Bruce, Kim. B. (2010): “On reacting to assertions and polar questions”. Journal of semantics 27/1: 81-118.

Féry, Caroline (2010): "Information Structure of schon". In: Hanneforth, Thomas/Fanselow, Gisbert (eds.) Language and Logos. A Festschrift for Peter Staudacher. Berlin, Akademieverlag: 160-175.

Forster, Kenneth. I./Forster, Jonathan. C. (2003): "DMDX: A Windows display program with millisecond accurancy". In: Behavior Research Methods, Instruments, \& Computers 35(1): $116-124$. 
Frey, Werner (1993): Syntaktische Bedingungen für die semantische Interpretation. Berlin: Akademie Verlag. (= Studia Grammatica 35)

Frey, Werner. (2000): "Syntactic requirements on adjuncts". ZAS papers in linguistics 17: 107-134.

Frey, Werner (2003): "Syntactic conditions on adjunct classes". In: Lang, Ewald et al. (eds.): Modifying adjuncts. Berlin, Mouton de Gruyter: 163-209.

Frey, Werner/Pittner, Karin (1998): „Zur Positionierung der Adverbiale im deutschen Mittelfeld“. Linguistische Berichte 176: 489-534.

Guillaume, Gustave (1964/1969) : Langage et science du langage. Quebec: Presses de l'Université Laval.

Gunlogson, Christine (2008): “A question of commitment”. Belgian Journal of Linguistics 22/1: 101-136.

Guy, Gregory. R. (2014): "Linking usage and grammar: Generative phonology, exemplar theory, and variable rules". Lingua 142: 57-65.

Haider, Hubert. (2000): “Adverb placement - Convergence of structure and licensing”. Theoretical Linguistics 26: 95-134.

Hall, T. Alan (1999): "Phonotactics and the Prosodic Structure of German Function Words". In: Hall, T. Alan/Kleinhenz, Ursula (eds.) Studies on the Phonological Word. Amsterdam/Philadelphia, Benjamins: 99-133. (Current Issues in Linguistic Theory 174.)

Herbst, Thomas (1996): “What are collocations: Sandy beaches or false teeth?" English Studies 77/4: 379-393. https://doi.org/10.1080/00138389608599038.

Hewson, John (2012): “Tense”. In: Binnik, Robert I. (ed.) The Oxford handbook of tense and aspect. Oxford, New York. Oxford University Press: 507-535.

Hinterhölzl, Roland (2006): Scrambling, Remnant Movement, and Restructuring in West Germanic. Oxford, New York: Oxford University Press. (= Oxford Studies in Comparative Syntax).

Hoepelman, Jakob/Rohrer, Christian (1981): "Remarks on noch and schon in German". In: Tedeschi, Philip/Zaenen, Annie (eds.), Syntax and Semantics 14: Tense and Aspect. New York, Academic Press: 103-126.

Jurafsky, Dan (2003): "Probabilistic Modeling in Psycholinguistics: Linguistic Comprehension and Production”. In: Bod, Rens/Hay, Jennifer/Jannedy, Stefanie (eds.) Probabilistic Linguistics: 39-96. Cambridge, MA MIT Press.

Keenan, Edward/Stabler, Edward (2003): Bare Grammar: Lectures on Linguistic Invariants. Chicago: CSLI.

Kentner, Gerrit (2017): "Rhythmic parsing." The Linguistic Review 34/1. DOI: 10.1515/ttr-20160005.

Klein, Wolfgang (1998). “Assertion and finiteness". In: Dittmar, Norbert/Penner, Zvi (eds.), Issues in the theory of language acquisition: Essays in honor of Jürgen Weissenborn. Bern, Lang: 225-245.

Klein, Wolfgang (2000): “An analysis of the German perfekt”. Language 76: 358-382.

Klein, Wolfgang (2006): About the German particles schon and noch. Ms. Nijmegen.

König, Ekkehard (1977): “Temporal and non-temporal uses of 'noch' and 'schon' in German". Linguistics and Philosophy 1.2: 173-198. 
Kutscher, Anna (2016): Optimale Adverbpositionen an der Schnittstelle zwischen Semantik, Phonologie und Syntax. Bielefeld: Universität Bielefeld.

Löbner, Sebastian (1989): "German schon - erst - noch: an integrated analysis". Linguistics and Philosophy 12: 167-212.

Löbner, Sebastian (1999): "Why German schon and noch are still duals: A reply to van der Auwera". Linguistics and Philosophy 22/1: 45-107.

Manning, Christopher (2003): "Probabilistic Syntax". In: Bod, Rens/Hay, Jennifer/Jannedy, Stefanie (eds.) Probabilistic Linguistics. 289-342. Cambridge, MA. MIT Press.

Mittwoch, Anita (1993): "The relationship between schon/already and noch/still: A reply to Löbner". Natural language semantics 2/1: 71-82.

Müller, Gereon (1995): A-bar Syntax: A Study of Movement Types. Berlin/New York: Mouton de Gruyter. (= Studies in Generative Grammar 42).

Müller, Gereon (1999): “Optimality, word order, and markedness in German”. Linguistics 37: 777-818.

Müller, Sonja (2018): Distribution und Interpretation von Modalpartikel-Kombinationen. www.oapen.org/download?type=document\&docid $=1001676$ [09.09.2019]

Musan, Renate (2002): The German perfect: its semantic composition and its interactions with temporal adverbials. Dordrecht/Boston/London: Kluwer Academic Publishers. (= Studies in Linguistics and Philosophy, 78)

Ormelius-Sandblom, Elisabet (1996): Die Modalpartikeln ja, doch und schon. Zur ihrer Syntax, Semantik und Pragmatik. Lund: Almqvist \& Wiksell International. (= Lunder germanistische Forschungen 61).

Pierrehumbert, Janet. B. (2006): "The next toolkit". Journal of Phonetics 34: 516-530.

Pollock, Jean-Yves (1989): "Verb movement, universal grammar, and the structure of IP". Linguistic inquiry 20/3: 365-424.

Primus, Beate (1994): „Grammatik und Performanz: Faktoren der Wortstellungsvariation im Mittelfeld“. Sprache und Pragmatik 32: 39-86.

Pustejovsky, James (2011): "Coercion in a general theory of argument selection". Linguistics 49/6: 1401-1431. doi:10.1515/ling.2011.039

Rathert, Monika (2012). “Adverbials". In: Binnik, Robert I. (ed.) The Oxford handbook of tense and aspect. Oxford, New York. Oxford University Press: 237-268.

Rosengren, Inger (2000): "Rethinking the Adjunct". In: Approaching the grammar of adjuncts: Proceedings of the Oslo Conference: 217-240.

Sabel, Joachim (2005): "String-vacuous scrambling and the Effect on Output Condition". In: Sabel, Joachim/Saito, Mamoru (eds.), The Free Word Order Phenomenon: Its Syntactic Sources and Diversity Berlin/New York, de Gruyter: 281-334. (= Studies in Generative Grammar 69).

Saffran, Jenny et al. (1996): "Statistical cues in language acquisition: Word segmentation by infants". In: Cottrell, Garrison W. (ed.) Proceedings of the 18th annual conference of the Cognitive Science Society, Hillsdale, NJ: 376-380.

Selkirk, Elizabeth (1996): "The prosodic structure of function words". In: Morgan, James/Demuth, Katherine (eds.) Signal to syntax: Bootstrapping from speech to grammar in early acquisition: 187-214. http://ifa.amu.edu.pl/ grzegorz/egg2009papers/intro/Selkirk_Function_Words.pdf [06.11.2019] 
Selkirk, Elizabeth (2011): The Syntax-Phonology Interface. In Goldsmith, John/Riggle, Jason/Yu, Alan (eds.): The Handbook of Phonological Theory. 2nd edition. Oxford: Blackwell.

Shih, Stephanie et al. (2015): "Rhythm's role in genitive construction choice in spoken English". In: van de Vijver, Ruben/Vogel, Ralf (eds.), Rhythm in cognition and grammar: 207-234.

Shukla, Mohinish/Nespor, Marina (2010): "Rhythmic patterns cue word order." In: ErteschikShir, Nomi/Rochman, Lisa (eds.): The sound patterns of syntax, New York: Oxford University Press: $174-188$.

Stalnaker, Robert (2002): “Common ground". Linguistics and philosophy 25/5: 701-721.

Stefanowitsch, Anatol/Gries, Stefan Th. (2003): "Collostructions: Investigating the interaction of words and constructions." In: International Journal of Corpus Linguistics 8/2: 209-243. https://doi.org/10.1075/ijcl.8.2.03ste.

Struckmeier, Volker (2014): Scrambling ohne Informationsstruktur? Prosodische, semantische und syntaktische Faktoren der deutschen Wortstellung. Berlin/Boston: de Gruyter. (= Studia Grammatica 77).

Valin, Roch (1994): L'envers des mots: analyse psychomécanique du langage. Sainte-Foy, Québec: Presses de l'Université Laval.

Van Der Auwera, Johan (1993): 'Already' and 'still': beyond duality. Linguistics and philosophy: 613-653.

Vogel, Ralf et al. (2015): "Function Words in Rhythmic Optimisation". In: van de Vijver, Ruben/Vogel, Ralf (eds.), Rhythm in Cognition and Grammar. A Germanic Perspective. Berlin: De Gruyter: 253-274.

Wiese, Richard (1996): The phonology of German. Oxford: Clarendon Press.

Wunderlich, Dieter (1970): Tempus und Zeitreferenz im Deutschen. München: Hueber

Wurmbrand, Susi (2008): "Word order and scope in German". Groninger Arbeiten zur Germanistischen Linguistik 46: 8-110

Zubizarreta, Maria (1998): Prosody, focus, and word order. Cambridge, Mass.: MIT Press (= Linguistic inquiry monographs 33).

\section{Anhang - Liste der getesteten Stimuli}

Sätze als Gesprächsausschnitte

1. „Universität“

- Wie läuft's mit der Ausbildung der Kinder, alle noch gut dabei?

- Konstanze ging immer schon fleißig zur Schule, zur Uni hingegen geht sie jetzt kaum noch.

2. „Mode“

- Was hat die denn an, soll das hübsch sein?

- Beate trug schon immer schräge Klamotten, doch dieses Ensemble ist einfach nur Schrott.

3. „Übervorsichtig““

- Hat sie Angst vor dem Straßenverkehr, oder was ist da los?

- Simone fuhr schon immer vorsichtig Fahrrad, jetzt hat sie 'nen Fahrradhelm auch noch dazu.

4. „Reifes Obst“

- Soll ich die Bananen wegschmeißen oder isst sie jemand in der WG? 
- Sabine aß immer schon reife Bananen, doch diese hier sind bereits ihr schon zu krass.

5. „Gehirnerschütterung“"

- Gerade ist ein Vogel voll gegen die Scheibe geflogen!

- Der Vogel sitzt immer noch auf dem Geländer, ihm geht es womöglich nicht unbedingt gut.

6. „Erinnerungsstücke“

- Warum schmeißt deine Mutter die alten Sachen nicht weg?

- Es waren Geschenke. Die Vase steht noch immer in der Vitrine, auch wenn sie bereits völlig unbrauchbar ist.

7. „Klemptner“

- Ist Ihr Wasserproblem jetzt beseitigt?

- Nein! Das Wasser tropft noch immer aus dem Behälter, obwohl diese Dichtung doch neu sein soll.

8. „Katzen“

- Eigentlich schlafen Katzen nur in der Sonne.

- Schau: Der Kater pennt immer noch auf der Terrasse, auch wenn dort die Sonne schon lange weg ist.

9. „Zugfahrt“"

- Ich muss heute unbedingt Katja den Ordner zukommen lassen. Wie mache ich das am cleversten?

- Marina wird heute noch Katja begegnen, sie fahren doch beide im gleichen Zug mit.

10. „Familienstreit“"

- Heute mal wieder Streit im Hause Meyer?

- Wie immer: Anita wird noch heute Thomas beschimpfen, weil er ihren Laptop kaputt gemacht hat.

11. „Autoreparatur“"

- Schafft es Gesine überhaupt zur Party in der Stadt? Ihr Auto ist doch kaputt?

- Klar, Gesine wird noch heute Lukas bestechen, damit er den Wagen für sie repariert.

12. „Besuch“

- Haben die Kinder nach dem Umzug noch Kontakt zu den alten Schulkameraden?

- Ja, schon. Tatjana wird heute noch Olga besuchen, weil sie ihre Freundin so selten sieht.

13. ,Konzert“"

- Weißt du, ob Dirk und Co. in der Halle auftreten?

- Die Maja hat oft schon den Dirk angerufen, doch leider war immer die Leitung besetzt.

14. „WG-Leben“

- Können sich deine Mitbewohner nicht ausstehen, oder was war das gerade?

- Die Sonja hat schon oft den Robert veralbert, doch manchmal zahlt er es ihr auch schon heim.

15. „Streit“

- Warum reden denn deine Schwestern jetzt nicht mehr miteinander?

- Die Anja hat schon oft die Klara beleidigt, doch diesmal ging alles ein Tacken zu weit.

16. „Abgewiesen“

- Sind die beiden ein Paar oder nur Freunde?

- Der Sascha hat oft schon die Lisa umworben, doch leider hat sie keine Lust auf ihn.

17. „Einschulung“ 
- Hat der Musikkurs was gebracht in Sachen Gesang?

- Wenig. Der Junge wird bald schon die Schule besuchen, nur ist er noch immer nicht wirklich begabt.

18. „Abschluss“

- Deine Nichte sieht aber auch sehr gestresst aus, was hat sie?

- Das Mädel wird schon bald die Prüfung bestehen, doch hat sie noch immer kein Studienplatz.

19. „Grillfest“

- Kommt der Geruch von den Nachbarn? Das stinkt ja fürchterlich

- Schau mal! Die Flamme wird schon bald die Würstchen verbrennen, nur kümmert sich keiner tatsächlich darum.

20. „Am Imbissstand“

- Hallo! Habe gerade kaum Zeit zum Warten. Kannst du mir was geben, was gleich fertig ist?

- Der Chefkoch hat bald schon die Burger gebraten, nur schmecken sie immer so richtig nach Müll.

21. „Auf dem Bauernhof“

- Schafft ihr es morgen rechtzeitig die Tiere zu versorgen?

- Der Peter hat jetzt schon die Ziegen gefüttert, und hat dadurch richtig viel Zeit eingespart.

22. „Am Telefon“

- Wie lange, tippst du, geht noch das Konzert?

- Nicht lange: Der Sänger hat schon jetzt das Lied abgebrochen; die Stimme versagt ihm bei jedem Refrain.

23. „Überfall“

- Gibt es neue Infos von der Polizei?

- Der Räuber hat schon jetzt 10 Leute bestohlen und ist leider immer noch auf freiem Fuß.

24. „Hochzeit“"

- Warum hast du was gegen die Hochzeit?

- Der Priester hat jetzt schon das Pärchen getraut, auch wenn die Verlobung erst vorgestern war.

25. „Gartenpflege“

- Der Park ist ja wirklich schön!

- Ein Gärtner pflegt den Park schon immer, deshalb sieht der auch gut aus.

26. „Gastauftritt““

- Wie kommt's, dass Madonna Like a virgin gesungen hat? Das passt gar nicht in die Show!

- Die Diva singt den Song immer schon, das ist ihr aller größter Hit.

27. „Langzeitpatient““

- Warum fährt Dr. Meyer so weit zu einem Hausbesuch?

- Der Doktor pflegt den Mann immer schon, das ist ein Langzeitpatient.

28. ,Weinprobe“"

- Der Wein sieht komisch aus, kann man den überhaupt trinken?

- Der Winzer trinkt den Wein schon immer, den stellt er auch selber her.

29. „Diebstahl“

- Warum ist Paul nicht vorzeitig entlassen worden?

- Der Richter straft den Dieb noch immer, damit es nicht nochmal passiert. 
30. ,Rentenalter“

- Will dein Vater nicht langsam in Rente gehen?

- Der Makler liebt den Job immer noch, weil er da gutes Geld verdient.

31. „Banküberfall““

- Gibt's was Neues über das gestohlene Geld?

- Die SoKo sucht das Geld immer noch, obwohl der Täter jetzt schon sitzt.

32. ,Fußballspiel“"

- Spielt dein Großvater noch Fußball?

- Der Opa trifft das Tor noch immer, dabei ist er doch beinah blind.

33. „Versicherung“"

- Müssen wir jetzt auch noch Folgeschäden übernehmen?

- Der Rücken schmerzt der Frau noch heute, der Unfall selbst ist Jahre her.

34. „Vatikan“

- Der Papst erklärte, dass mit den Finanzen alles in Ordnung sei.

- Die Kirche glaubt dem Papst heute noch, die Fakten sehen anders aus.

35. „Büroalltag“

- War das eben die Krüger im Fahrstuhl?!

- Die Tipse stalkt den Chef heute noch, obwohl sie längst gefeuert ist.

36. ,Tierquäler“

- Die Bauernhöfe hier sind mittlerweile alle auf dem neuesten Stand.

- Denkste! Die Stute zieht den Pflug noch heute, auch wenn der Sepp 'nen Traktor hat.

37. „Urlaub“

- Verbringt Marcus seinen Urlaub wieder am Mount Everest?

- Neee. Den Berg bestiegen hat er oft schon, jetzt will er auch mal segeln gehen.

38. „Seven King“

- Warum will Lars unbedingt Es im Kino sehen?

- Den King gelesen hat er schon oft, jetzt will er die Verfilmung sehen.

39. ,Seitensprung“

- Warum hat Jana denn den Seitensprung gebeichtet?

- Den Mann betrogen hat sie schon oft, nun will sie endlich ehrlich sein.

40. ,Computer“

- Claudia muss doch nicht alles per Hand eintragen, da gibt's 'ne App für?

- Die App verwendet hat sie oft schon, nur stürzt seitdem der Browser ab.

41. „Bei der Lesung“"

- Wie weit ist Jörg mit den Vorbereitungen? Ist die Location schon soweit?

- Den Saal bestuhlen wird er bald schon, dann kann da keiner weiter rein.

42. „Geburtstag““

- Wir sollten mal wieder mit Christoph was trinken gehen.

- Ein Fest bereiten wird er schon bald, denn sein Geburtstag steht bevor.

43. „Konkurrenz“

- Hat Tim ein gutes Verhältnis zu seinem Bruder?

- Noch: Den Tom beneiden wird er schon bald, denn dieser hat den besseren Job.

44. „Renovierung“

- Will Stephan das gesamte Haus noch vor dem Sommer umbauen? 
- Das Dach zerlegen wird er bald schon, weil das doch jetzt schon undicht ist.

45. „Lagerfeuer“

- Braucht man trockeneres Holz für das Feuer?

- Den Brand entfachen wird es jetzt schon, die Hitze ist schon stark genug. 46. „Malerarbeiten“

- Darf Alex die Fenster aufmachen, wenn die Farbe noch trocknet?

- Den Raum entlüften muss er schon jetzt, sonst stinkt das ganze Haus danach. 47. „Grillfest“"

- Andreas will morgen den Abwasch erledigen, heute will er nicht mehr.

- Das Rost entfetten muss er schon jetzt, sonst trocknet das später unglaublich fest.

48. ,Marmelade kochen

- Soll Mascha einfach alles in den Topf schmeißen und kochen?

- Das Obst entkernen muss sie jetzt schon, beim Kochen der Früchte stört der Kern nur. 